\title{
Transcriptomic profiles of aging in purified human immune cells
}

Lindsay M Reynolds', Jingzhong Ding ${ }^{2}$, Jackson R Taylor ${ }^{3}$, Kurt Lohman $^{4}$, Nicola Soranzo ${ }^{5}$, Alberto de la Fuente ${ }^{6}$, Tie Fu Liu ${ }^{2}$, Craig Johnson ${ }^{7}$, R Graham Barr ${ }^{8}$, Thomas C Register ${ }^{9}$, Kathleen M Donohue ${ }^{8}$, Monica V Talor ${ }^{10}$, Daniela Cihakova ${ }^{10}$, Charles Gu ${ }^{11}$, Jasmin Divers ${ }^{4}$, David Siscovick ${ }^{12}$, Gregory Burke ${ }^{1}$, Wendy Post ${ }^{10}$, Steven Shea ${ }^{8}$, David R Jacobs Jr ${ }^{13}$, Ina Hoeschele ${ }^{14}$, Charles E McCall ${ }^{2,15}$, Stephen B Kritchevsky ${ }^{2,3}$, David Herrington ${ }^{2}$, Russell P Tracy ${ }^{16}$ and Yongmei Liu ${ }^{1 *}$

\begin{abstract}
Background: Transcriptomic studies hold great potential towards understanding the human aging process. Previous transcriptomic studies have identified many genes with age-associated expression levels; however, small samples sizes and mixed cell types often make these results difficult to interpret.

Results: Using transcriptomic profiles in CD14+ monocytes from 1,264 participants of the Multi-Ethnic Study of Atherosclerosis (aged 55-94 years), we identified 2,704 genes differentially expressed with chronological age (false discovery rate, FDR $\leq 0.001$ ). We further identified six networks of co-expressed genes that included prominent genes from three pathways: protein synthesis (particularly mitochondrial ribosomal genes), oxidative phosphorylation, and autophagy, with expression patterns suggesting these pathways decline with age. Expression of several chromatin remodeler and transcriptional modifier genes strongly correlated with expression of oxidative phosphorylation and ribosomal protein synthesis genes. 17\% of genes with age-associated expression harbored $\mathrm{CpG}$ sites whose degree of methylation significantly mediated the relationship between age and gene expression $(p<0.05)$. Lastly, 15 genes with age-associated expression were also associated (FDR $\leq 0.01$ ) with pulse pressure independent of chronological age. Comparing transcriptomic profiles of CD14+ monocytes to CD4+ T cells from a subset $(n=423)$ of the population, we identified 30 age-associated (FDR < 0.01) genes in common, while larger sets of differentially expressed genes were unique to either T cells (188 genes) or monocytes (383 genes). At the pathway level, a decline in ribosomal protein synthesis machinery gene expression with age was detectable in both cell types.

Conclusions: An overall decline in expression of ribosomal protein synthesis genes with age was detected in CD14+ monocytes and CD4+ T cells, demonstrating that some patterns of aging are likely shared between different cell types. Our findings also support cell-specific effects of age on gene expression, illustrating the importance of using purified cell samples for future transcriptomic studies. Longitudinal work is required to establish the relationship between identified age-associated genes/pathways and aging-related diseases.
\end{abstract}

Keywords: Aging, Monocyte, T cell, Transcriptome, Mitochondrial ribosome, Translation, Protein synthesis, Ribonucleoprotein complex, Oxidative phosphorylation, Autophagy, Methylation

\footnotetext{
* Correspondence: yoliu@wakehealth.edu

'Department of Epidemiology and Prevention, Division of Public Health Sciences, Wake Forest School of Medicine, Winston-Salem, North Carolina 27157, USA

Full list of author information is available at the end of the article
} 


\section{Background}

Identifying molecular features that vary with chronological age has critical implications for our understanding of aging and the development of age-associated diseases. A number of previous studies have performed systematic investigations of the relationship between age and gene expression in various human tissues, including $\mathrm{T}$ cells [1-3], whole blood [4], peripheral blood mononuclear cells (PBMCs) [5], brain [6-8], and muscle tissue $[7,9]$. Although very few individual genes with ageassociated expression have been identified across studies or tissues [8], similar gene functions/pathways have been reported. For instance, pathway analyses of age-associated genes identified an enrichment of immune function and inflammation genes in various mixtures of blood cells [2-5]. Other aging-associated pathways/processes found to be enriched in blood as well as brain and muscle tissues include RNA processing $[6-8,10]$ and chromatin remodeling $[6,7,10]$, while mitochondrial pathways $[6,8]$ and more specifically the oxidative phosphorylation/electron transport pathway $[7,9]$ were detectable in studies of skin, brain, and muscle tissues. However, interpretation of these findings is limited by small sample sizes and often heterogeneous cellular composition of the samples investigated. Currently, there is a lack of well-powered transcriptomic studies of aging using homogeneous cell samples.

CD4+ T cells and CD14+ monocytes are excellent cell types for transcriptomic studies of aging in humans. $\mathrm{T}$ cells and monocytes can be isolated from an easily accessible tissue (blood), and both have known roles in the development of age-related diseases. $\mathrm{T}$ cells are well known to exhibit numerous functional impairments with advanced age and have been implicated in the agedependent decline in immune function, commonly known as immunosenescence [11]. To date, the largest aging transcriptomic study of CD4+ T cells included 31 individuals, aged $25-81$ years, from the Baltimore Longitudinal Study of Aging. Comparison of T cell expression profiles from individuals less than 65 years of age to those age 65 and older revealed 264 genes associated with age $(\mathrm{p}<0.05$, FDR < 0.3) [3]. Monocytes have also been shown to exhibit phenotypic and functional changes in the elderly [12], and are key cells of innate immunity and major contributors to the pathogenesis of inflammatory and degenerative diseases [13]. To our knowledge the effect of aging on the monocyte transcriptome has not yet been investigated.

Previously, we purified CD14+ monocytes from the peripheral blood of 1,264 participants of the MultiEthnic Study of Atherosclerosis (MESA). We measured both genome-wide gene expression and DNA methylation in these purified monocyte samples using microarrays, and identified cytosine-guanine dinucleotides (CpGs) whose degree of methylation was associated with cis-gene expression (FDR < 0.001) [14]. Given that DNA methylation can vary with age [15-20], we also investigated the relationship between age and DNA methylation in the 1,264 MESA monocyte samples [21], and identified 1,794 CpGs whose degree of methylation was associated with age and cis-gene expression (FDR < 0.001) [21]. However, it remains unknown to what extent age-related variations in the methylome may mediate the overall effect of age on gene expression.

In response to these uncertainties, here we present a comprehensive analysis of age and gene expression in the CD14+ monocyte samples from 1,264 MESA participants ranging in age from 55 to 94 years. Additionally, we present an analysis of age and gene expression in circulating $\mathrm{CD} 4+\mathrm{T}$ cells from a subset of 423 MESA participants. This cohort study offers the unique opportunity to 1 ) better understand the effect of aging on gene expression in CD14+ monocytes and CD4+ T cells, 2) compare the aging transcriptome measured in two cell types from the same individuals, 3) investigate $\mathrm{CpG}$ methylation as a potential mediator of age-associated variations in the transcriptome, and 4) characterize the relationships between chronological age-associated gene expression and a clinical measure of vascular age, pulse pressure.

\section{Results and discussion}

The overall study design and results are summarized in Figure 1, and the population characteristics are described in Additional file 1: Table S1.

\section{Transcriptomic profiles associated with age in 1,264 monocytes samples}

Transcriptomic profiling of CD14+ monocytes using microarrays (Illumina HumanHT-12 v4 Expression BeadChip) revealed 10,898 expressed genes, of which $25 \%$ had expression significantly (FDR $\leq 0.001, \mathrm{p}<9.0 \times 10^{-4}$ ) associated with chronological age (Figure 1A, and Additional file 1: Figure S1) after adjusting for appropriate biological and technical covariates including race, gender, study site, and estimates of residual sample contamination with non-targeted cell types (see Methods). The effect size of a ten-year difference in age for individual gene expression was modest (up to $10 \%)$. The majority of the associations with age remained significant in the "disease free" (no report of diabetes, cancer, or cardiovascular diseases; $n=839$ ), sex- and ethnicspecific subgroups (Additional file 2: Table S2).

Gene set enrichment analysis [22,23] identified the ribonucleoprotein complex, mitochondrial ribosome, and oxidative phosphorylation pathway genes enriched among age-associated genes. After stratifying by the effect direction of age on expression, the genes with expression negatively associated with age were found to be enriched with ribosomal/translation and mitochondrial/oxidative phosphorylation genes (Additional file 1: Table S3). In contrast, 


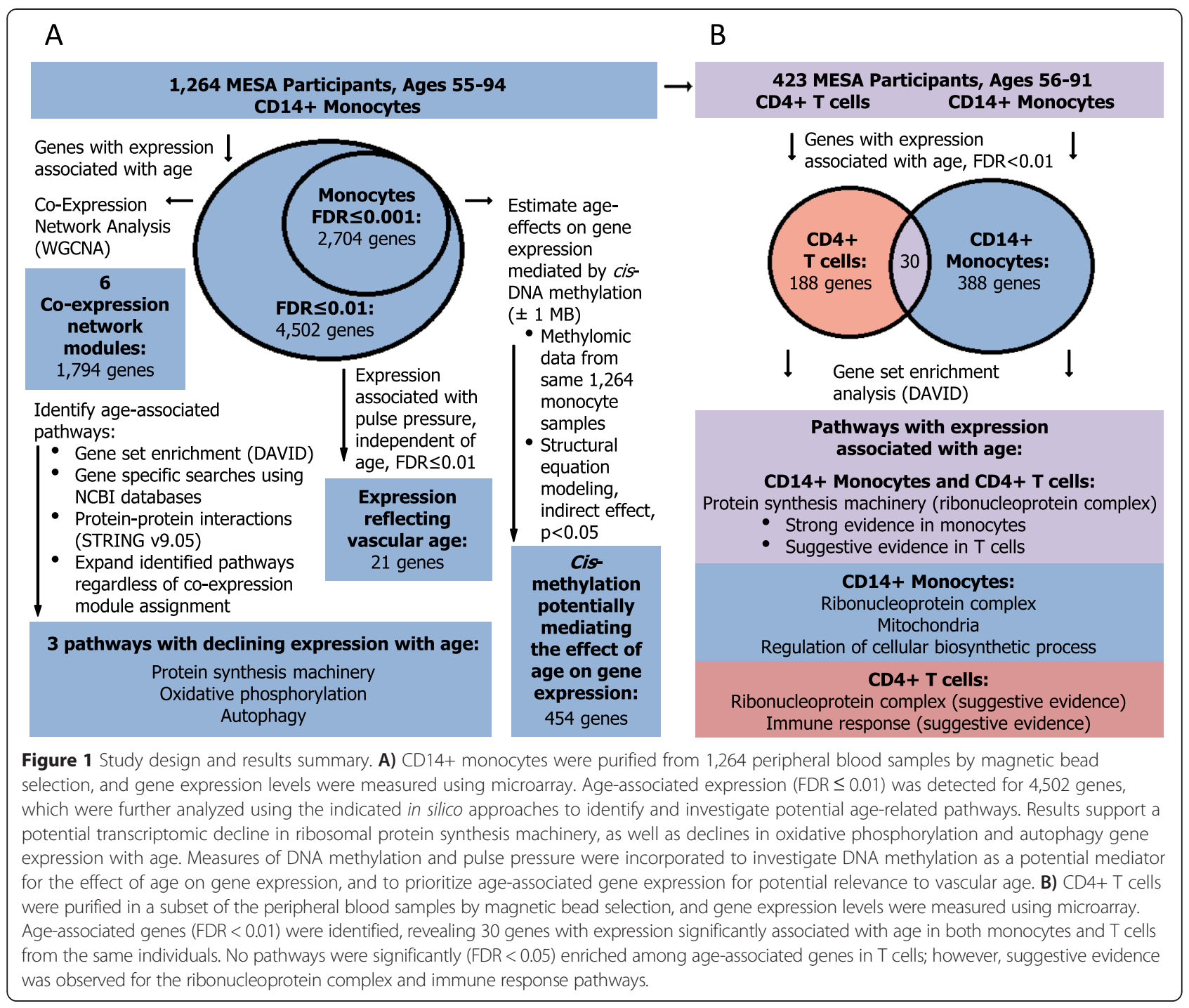

genes with expression positively associated with age were enriched with pathways relating to regulation of transcription, the cytoskeleton, protein phosphorylation, and response to insulin.

\section{Co-expression network analysis}

To identify consensus networks of genes with coordinated expression profiles associated with age, we used a weighted gene co-expression network analysis [24] (WGCNA), combined with a stability analysis (see Methods), and examined an expanded set of 4,502 genes associated with age at a more liberal FDR threshold of $\leq 0.01$ (Figure $1 \mathrm{~A}$ and Additional file 2: Table S2). Six mutually exclusive gene network modules were identified, labeled as colors: 'black', 'blue', 'turquoise', 'brown', 'yellow', and 'green'. Network modules ranged in size from three to 1,466 genes, and had significant module eigengene (1st eigenvector) associations with age with $\mathrm{p}$ ranging from $1.8 \times 10^{-30}$ to $1.3 \times 10^{-6}$ (Figure 2 and Additional file 1 :
Table S4). Genes assigned to the same module had moderately to strongly correlated expression (absolute median $\mathrm{r}$ ranging $0.41-0.62$ ). To better understand the relationships between modules, we examined the correlations between eigengenes of each module, and found a very strong negative correlation between the 'blue' and 'turquoise' module eigengenes (correlation $=-0.90$; Additional file 1: Figure S2).

Gene set enrichment analysis revealed significantly $(F D R<0.05)$ enriched pathways within two of the six modules, relative to all monocyte expressed genes (Table 1). The 217 genes assigned to the 'blue' module, all with expression negatively associated with age, were enriched with 1) ribonucleoprotein complex genes (including translation, ribosome biogenesis, and RNA processing genes) and 2) mitochondrion genes (including oxidative phosphorylation and mitochondrial ribosome genes). The largest gene network module, the 'turquoise' module, was assigned 1,466 genes which were significantly enriched with nuclear 


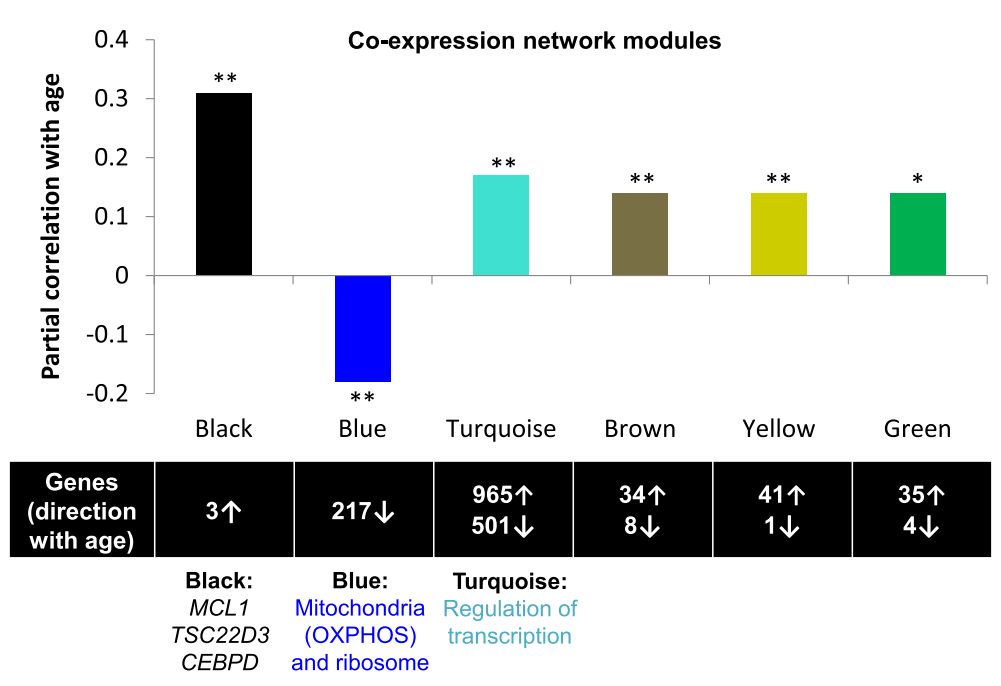

Figure 2 Co-expression network modules associated with chronological age. Six mutually exclusive gene network modules with coordinate gene expression profiles associated with chronological age were identified in 1,264 monocyte samples (using WGCNA), ranging in size from 3 to 1,466 genes. For each module (x-axis), the partial correlation between age and the module eigengene is shown (y-axis); covariates included race, sex, site of data collection, and residual sample contamination with non-targeted cells. Below each module is the number of genes assigned to the module, and the direction of expression association with age; network modules discussed in further detail include the 'black' module (containing three genes: MCL1, TSC22D3, and CEBPD), and the 'blue' and 'turquoise' modules (which were significantly enriched with age-related pathways shown in Table 1). The significance of the module eigengene association with age is denoted as: ${ }^{*} p<0.008$ (Bonferroni adjusted significance threshold for testing six modules, alpha $=0.05)$, and ${ }^{* *} p \leq 1 \times 10^{-6}$.

lumen genes, many of which with known roles in RNA splicing and the regulation of transcription.

The other four co-expression network modules (shown in Figure 2 as 'black', 'brown', 'yellow', and 'green') were found to have weakly to moderately correlated eigengenes (Additional file 1: Figure S2) which were positively associated with older age. No significantly enriched pathways were detected within these four modules with a false discovery rate of $5 \%$; however, there were pathways enriched with nominal significance among these modules, including insulin signaling ('brown', fold enrichment $=19.9, \mathrm{p}=8.88 \times 10^{-4}$ ), immune response ('yellow', fold enrichment $=5.1, \mathrm{p}=1.97 \times 10^{-4}$ ), and regulation of apoptosis ('green', fold enrichment 3.9, $\left.\mathrm{p}=1.12 \times 10^{-3}\right)$.

\section{Autophagy-related gene expression}

Autophagy is a degradation pathway that utilizes double-membrane vesicles, termed autophagosomes, to engulf long-lived proteins, damaged organelles, and

Table 1 Significantly enriched Gene Ontology terms among co-expression network modules in CD14+ monocytes

\begin{tabular}{|c|c|c|c|c|c|}
\hline Gene Ontology term & Gene Count & Direction of age effect & Fold Enrichment & Nominal P-value & FDR \\
\hline \multicolumn{6}{|c|}{ 'Blue' network module (217 genes; $217 \downarrow 0 \uparrow)$} \\
\hline Ribonucleoprotein complex: & 58 & $(58 \downarrow 0 \uparrow)$ & 5.6 & $5.71 \mathrm{E}-29$ & 7.40E-26 \\
\hline Translation & 41 & $(41 \downarrow 0 \uparrow)$ & 6.9 & $3.48 \mathrm{E}-23$ & 5.20E-20 \\
\hline Ribosome biogenesis & 16 & $(16 \downarrow 0 \uparrow)$ & 7.1 & 4.57E-09 & $6.84 \mathrm{E}-06$ \\
\hline RNA processing & 26 & $(26 \downarrow 0 \uparrow)$ & 2.8 & $5.25 \mathrm{E}-06$ & 7.86E-03 \\
\hline Mitochondrion: & 61 & $(61 \downarrow 0 \uparrow)$ & 3.2 & $5.67 \mathrm{E}-18$ & 7.36E-15 \\
\hline Oxidative phosphorylation & 15 & $(15 \downarrow 0 \uparrow)$ & 10.0 & $1.57 \mathrm{E}-10$ & 2.35E-07 \\
\hline Respiratory chain complex I & 8 & $(8 \downarrow 0 \uparrow)$ & 11.3 & 4.55E-06 & 5.90E-03 \\
\hline \multicolumn{6}{|c|}{ Turquoise' network module (1,466 genes; $501 \downarrow 965 \uparrow)$} \\
\hline Nuclear lumen: & 199 & $(48 \downarrow 151 \uparrow)$ & 1.4 & $9.05 \mathrm{E}-07$ & $1.32 \mathrm{E}-03$ \\
\hline RNA splicing & 59 & $(17 \downarrow 42 \uparrow)$ & 1.8 & $1.43 \mathrm{E}-05$ & $2.54 \mathrm{E}-02$ \\
\hline
\end{tabular}

Results from gene set enrichment analysis are presented (from DAVID) for co-expression network modules with significantly enriched Gene Ontology (GO) terms $($ FDR < 0.05). No significant enrichment was observed among the 'black', 'brown', 'yellow', or 'green' network module genes; background genes included all 10,898 genes with expression detectable in 1,264 CD14+ monocyte samples.

$\uparrow$ genes with expression positively associated with age; $\downarrow$ genes with expression negatively associated with age. 
invasive pathogens, and to transport these cargos to the lysosomes for degradation [25]. In the aging field, impaired autophagy is considered one of the principal determinants of cellular aging, which is supported by in vitro and animal study findings that autophagy declines with age [26]. However, studies of autophagy and age in humans are sparse.

One of the most significant age-gene expression associations we observed in monocytes from 1,264 individuals was with $M C L 1$ (myeloid cell leukemia sequence 1; $\left.\mathrm{FDR}=7.6 \times 10^{-16}\right) . M C L 1$, a known inhibitor of autophagy and apoptosis, is a member of the Bcl-2 (B-cell CLL/ lymphoma 2) family, which includes many other proteins known to regulate autophagy and apoptosis [27-29]. The positive relationship between MCL1 expression and age tends to be linear across the range of ages (55 - 94 years) in this population (Additional file 1: Figure S3). We confirmed an age-associated increase in MCL1 mRNA expression in a subset of the population using RNA sequencing technology $\left(\mathrm{n}=373 ; \mathrm{p}=2.98 \times 10^{-5}\right.$; Additional file 1: Figure S4). MCL1 gene expression was also significantly correlated with MCL1 protein expression measured in a subset of the population using Western Blot for $(n=30, r=0.42$; $\mathrm{p}$-value $=0.02$; Additional file 1: Figure S5) .

MCL1 was assigned to the co-expression network module whose eigengene was most significantly associated with age ('black', peigengene $=1.79 \times 10^{-30}$ ). In addition to MCL1, the 'black' module contained two other genes with expression positively associated with age TSC22D3 (TSC22 domain family, member 3; FDR = $6.69 \times 10^{-24}$ ) and CEBPD (CCAAT/enhancer binding protein, delta; FDR $=3.82 \times 10^{-15}$ ), which encode transcription factors involved in the suppression of inflammation and apoptosis [30,31]. While a common regulator for these three 'black' module genes has not been identified, the limited literature available points towards cytokines such as IL-2 (Interleukin 2) and IL-6 in the upregulation of 'black' module gene expression, possibly through the activation of STAT proteins [30,32-34]. Notably, STATs 1, 3, 4, and 5A were also found in our list of genes that increase expression with age $(\mathrm{FDR}=$ $3.59 \times 10^{-6}, 5.40 \times 10^{-7}, 6.46 \times 10^{-5}$, and $2.49 \times 10^{-3}$, respectively).

Given the limitation of the WGCNA network analysis (hierarchical clustering only allows single module membership), and the known role for MCL1 in the inhibition of autophagy [29], we next examined the relationship between age and expression for key autophagy genes disregarding network module membership. The associations of age and gene expression, as well as the previously characterized protein-protein interactions [35], are shown for key autophagy genes in Figure 3. Among the well-known regulators of autophagy within the Bcl-2 family [36], age was positively associated with expression of inhibitors of autophagy (i.e. $M C L 1, B C L 2$, and $B C L 2 L 2$; FDR: $7.60 \times 10^{-16}-1.15 \times 10^{-3}$ ), and negatively associated with expression of activators of autophagy (i.e. $B A D$ and BID; FDR: $8.28 \times 10^{-7}$ and $1.18 \times 10^{-4}$, respectively). Negative effects of age on gene expression were also observed for genes which encode proteins critical for autophagosome formation [26], including autophagy machinery genes ATG3, ATG5, ATG7, and GABARAPL2 (FDR ranging $3.48 \times 10^{-4}-1.8 \times 10^{-3}$ ). Additionally, we observed a positive effect of age on the expression of autophagy inhibitors belonging to the PI3K/ Akt signaling pathway (MTOR, IL10RA, STAT3, JAK1, PDPK1, IL2RB; FDR ranging $1.45 \times 10^{-8}-9.88 \times 10^{-4}$ ), while negative effects of age were observed for a PI3K/Akt signaling pathway gene important for autophagy activation [37,38], AMPK (PRKAG1, FDR $\left.=4.87 \times 10^{-7}\right)$. However, exceptions to the apparent age-dependent transcriptional decline of autophagy gene expression were also observed, such as increasing expression of pro-autophagy genes [39], BECN1 (Beclin-1, autophagy related; FDR $=1.33 \times 10^{-4}$ ) and ULK1 (unc-51-like kinase 1; FDR $=9.97 \times 10^{-5}$ ) with older age.

The protein networks that regulate autophagy and apoptosis are highly interconnected, and crosstalk has been observed, particularly among Bcl-2 family members [36]. However, an overall transcriptional decline in apoptosis gene expression with age was not apparent, as other key regulators of the apoptotic pathway, such as pro-apoptotic CASP2, CASP8, and FOXO3, had increasing expression associated with older age $(F D R=$ $3.9 \times 10^{-4}, 4.5 \times 10^{-3}$, and $6.0 \times 10^{-8}$, respectively).

In vitro and animal studies have reported a decline in autophagy with age $[26,36,40-43]$; however, to our knowledge, only one other publication has reported an age-associated decline in expression of autophagy genes, which was carried out in a small number of human brain tissue samples [44]. Overall, these findings for major components of core autophagy machinery and upstream regulators provide evidence for a transcriptional decline in autophagy gene expression with age in human monocytes. The identification of key genes contributing to a decline in autophagy are of great interest, as pharmacologic activation of autophagy has been linked with increasing lifespan in animal models, including mice [45]. Further, dysfunctional autophagy is now widely implicated in pathophysiological processes of many age-related diseases such as cancer, Alzheimer's, diabetes, and cardiovascular diseases [46]. However, longitudinal studies are necessary to validate the age-related transcriptional decline of autophagy gene expression in human monocytes, and to investigate the relationship between these age-related patterns and the development of age-associated diseases. 


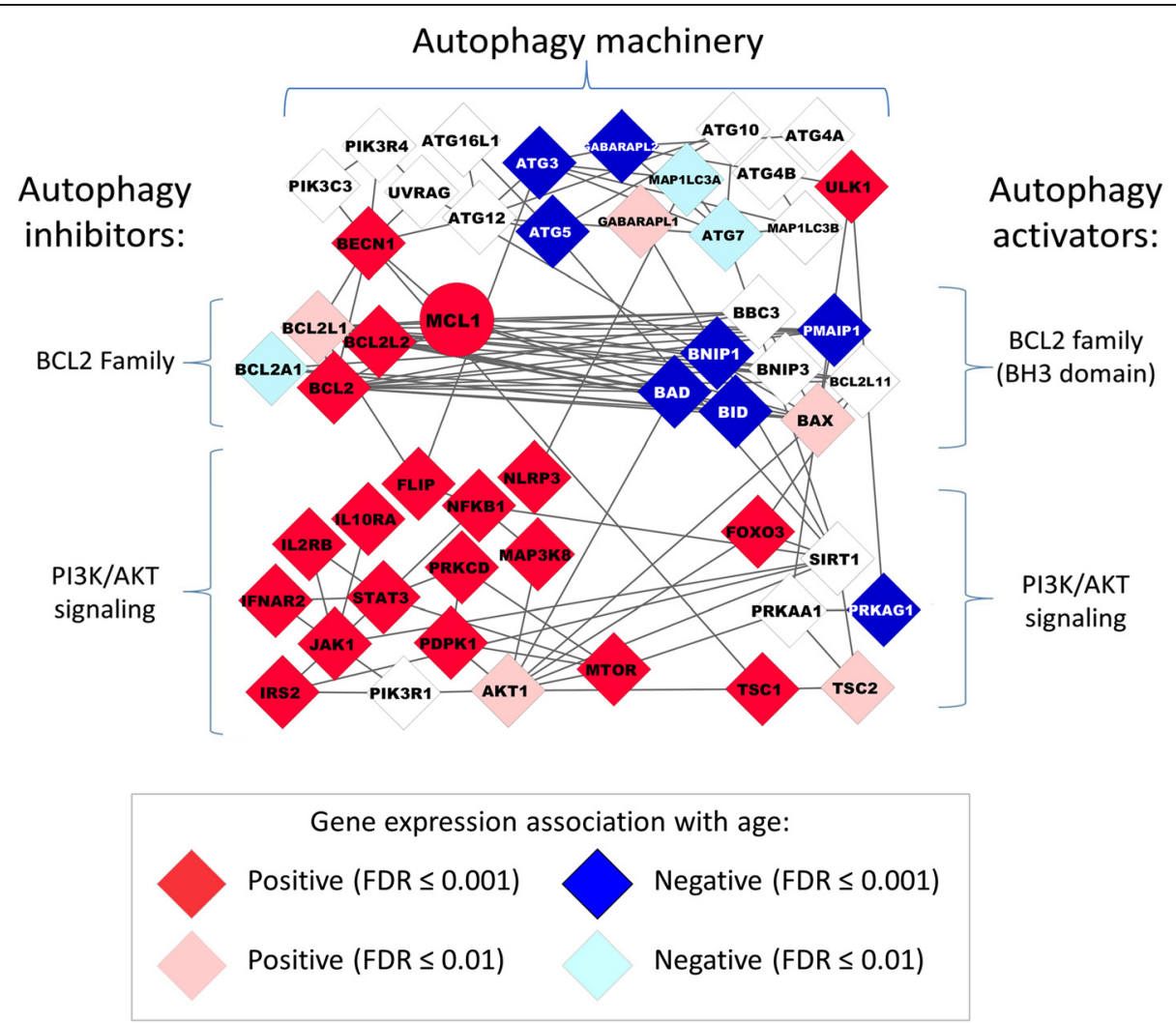

Figure 3 Age-associated expression pattern for the Bcl-2 family and other key autophagy genes suggest autophagy declines with age. The 'black' co-expression network module gene - MCL1 (circle), and other key genes (diamonds) encoding autophagy machinery and autophagy inhibitors/activators (related to the BCl-2 family and the PI3K/AKT signaling pathway) are shown, with edges representing previously characterized protein-protein interactions (STRING v9.05). Color denotes the direction and significance resulting from the association of age and gene expression in 1,264 CD14+ human monocyte samples, adjusting for race, sex, study site, and residual cell contamination with other cell types.

\section{Oxidative phosphorylation and protein synthesis machinery gene expression}

According to the mitochondrial theory of aging, mitochondria are among the key players contributing to the aging process, whose dysfunction is linked with aging [47] and age-related diseases [48,49]. Consistent with previous findings from multiple human tissues and across species [50], our data revealed a pattern of decreasing expression of mitochondrial oxidative phosphorylation (OXPHOS) genes with age in monocytes, particularly among genes within the 'blue' network module (Table 1). The 'blue' module genes were also enriched with ribonucleoprotein complex genes. Upon examining 'blue' module genes for previously characterized protein-protein interactions (Figure 4), two subnetworks were identified: one relating to the mitochondrial electron transport chain, and the other composed of ribonucleoprotein complex genes. The majority of the ribonucleoprotein complex genes were ribosomal protein synthesis machinery genes.

To better understand the relationship between age and global expression of OXPHOS and ribosomal protein synthesis genes, we examined the associations between age and expression of all OXPHOS (54 genes, GO:0006119), ribosome (204 genes, GO:005840), and mitochondrial ribosomal genes (51 genes, GO:0005761) expressed in monocytes, disregarding network module membership. Overall, we found almost two-thirds of the expressed OXPHOS genes (61\%) and ribosomal genes (67\%) had expression negatively associated with age (FDR $\leq 0.01$, Additional file 2: Table S5 and Additional file 2: Table S6). Among the mitochondrial ribosomal genes, $80 \%$ had expression negatively associated with age $(\mathrm{FDR} \leq 0.01)$ (Additional file 2 : Table S7). Using western blot to measure the protein levels of one mitochondrial ribosomal protein (MRPS12) in a subset of our samples $(n=28)$, we found protein levels of MRPS12 tended to correlate with mRNA expression levels $(\mathrm{r}=0.29, \mathrm{p}=0.14$; Additional file 1: Figure S6).

The declining expression of oxidative phosphorylation genes in monocytes is consistent with previous findings across species [50-53], and from previous transcriptomic studies of aging in human muscle [7,9], and brain [7] tissues. Mitochondrial dysfunctional has been widely reported with aging [54] and many age-related diseases 


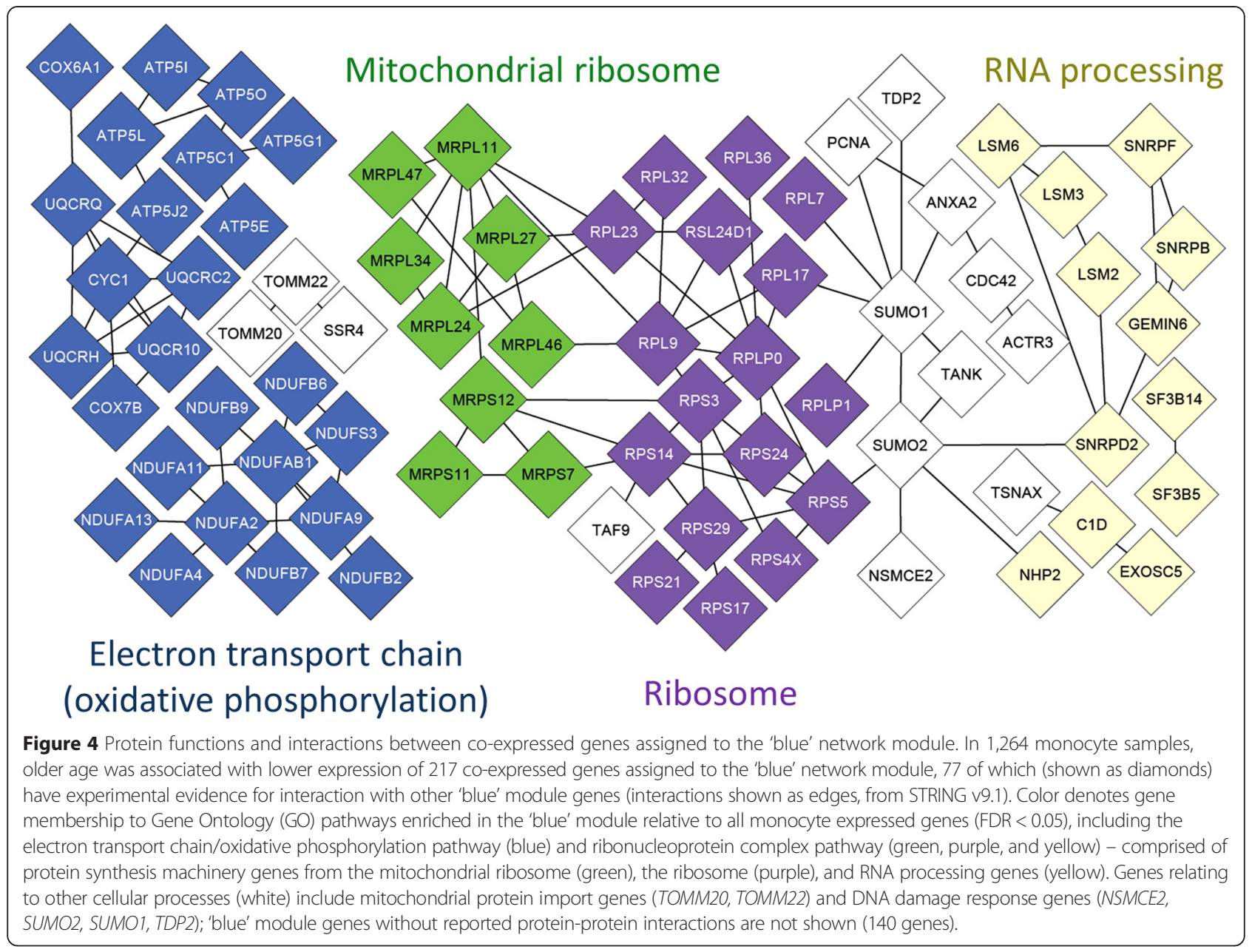

[49]. A major finding of this work is the coordinated down regulation of many oxidative phosphorylation and protein synthesis machinery genes with age in monocytes. However, the potential upstream mechanisms responsible for down-regulation of OXPHOS and protein synthesis genes with age are unclear.

\section{Potential drivers of an apparent age-related decline in} oxidative phosphorylation and protein synthesis machinery gene expression

Six genes with reported regulatory roles for mitochondrial gene expression [55,56] were detectable in our monocyte samples (PGC-1 $\alpha$, TFB2M, TFAM, MTERF, $N R F-2, P O L R M T$ ), two of which had expression significantly and negatively associated with age (TFB2M - transcription factor B2, mitochondrial, FDR $=9.94 \times 10^{-9}$; and $M T E R F$ - mitochondrial transcription termination factor, $\mathrm{FDR}=1.44 \times 10^{-4}$ ). However, there were no detectable transcriptional changes with age for PCG-1 $\alpha$, a master regulator of mitochondrial biogenesis, which indirectly up-regulates expression of nuclear OXPHOS genes, mitochondrial protein synthesis machinery, and mitochondrial protein import genes [57].

To identify potential transcriptional regulators for the coordinated expression of oxidative phosphorylation and protein synthesis machinery genes observed in monocytes, we next looked for enrichment [22] of transcription factor binding sites (TFBS) among genes assigned to the 'blue' co-expression network module. No TFBS were significantly $(F D R<0.05)$ enriched among 'blue' module genes compared to all monocyte expressed genes. The 'turquoise' module on the other hand, which was strongly and negatively correlated with the 'blue' module $(\mathrm{r}<-0.90$; Additional file 1: Figure S2), contained a large number of genes (1,466 genes) which were enriched with binding sites for over 50 different transcription factors compared to all monocyte expressed genes (Additional file 2: Table S8). Further, the 'turquoise' module included 238 genes with known roles in regulation of transcription (GO:0045449), including a number of transcription factors with expression increasing with older age (FOXO4, YY1, NFKB1, AHR; FDR ranging $1.48 \times 10^{-7}-$ 
6.50 $\times 10^{-4}$ ) and chromatin remodelers which increased with older age (SWI/SNF family genes: ARID1A, SMARCA4, SMARCA2, SMARCC2; FDR ranging $1.10 \times 10^{-5}$ $5.94 \times 10^{-4}$ ) (Additional file 2: Table S9). Future studies may benefit from our identification of several chromatin remodeler and transcriptional modifier genes with expression profiles strongly correlated with an apparent coordinated transcriptional decline of key genes required for mitochondrial biogenesis.

Paradoxically, inhibition of autophagy should reflect an anabolic state and increasing rates of protein synthesis and oxidative phosphorylation [58]; however, in the aging monocyte transcriptome we observed a potential transcriptional decline in autophagy, and a concomitant decline in OXPHOS and protein synthesis gene expression with age. Intriguingly, a decline in AMPK activity with age could potentially explain this paradox, given the dual role of AMPK to activate autophagy and mitochondrial biogenesis $[37,57,59]$. Similar to the decline in AMPK activity that has been previously reported in aged mice [54], here, we reported decreased expression of the regulatory subunit of AMPK (PRKAG1). These results provide clues for further investigations of the role of AMPK dysfunction in aging, and identify potential transcriptional regulators of an age-related decline in oxidative phosphorylation and ribosomal protein synthesis machinery gene expression.

Epigenomic regulation of age-associated gene expression To investigate DNA methylation as a potential regulator of the aging transcriptome, we performed lookups using the list of expression-associated methylation sites (eMS) that we recently reported from the same 1,264 monocyte samples [14]. We identified $48 \%$ of age-associated genes $(1,304$ genes, $F D R \leq 0.001)$ harboring eMS. Methylation profiles were both negatively correlated (69\%; range: $-0.77,-0.13$ ) and positively correlated (31\%; range: $0.13,0.73$ ) with gene expression profiles. Using mediation analyses to investigate DNA methylation as a potential mediator for the effect of age on gene expression, $17 \%$ of age-associated genes ( 454 genes, FDR $\leq 0.001$ ) were identified harboring $\mathrm{CpG}$ sites whose degree of methylation significantly mediated ( $\left.p_{\text {indirect }}<0.05\right)$ the effect of age on gene expression (Additional file 2: Table S10), including a similar percentage of genes from each of the three age-associated pathways: five OXPHOS genes (21\%), 18 ribosomal genes (18\%), and five autophagy genes (19\%) (Table 2). The majority of the mediation effects had similar directions of effect as the overall effect of age on gene expression (85\%).

Different from previous studies of the aging transcriptome that did not have measures of DNA methylation, our concurrent transcriptomic and methylomic profiling of the same batch of monocytes allowed us to detect genes harboring $\mathrm{CpG}$ sites with methylation profiles which significantly mediated the associations between age and gene expression. These potentially functional CpGs are enriched in predicted enhancer regions compared to all CpGs measured by microarray [14,21], suggesting that DNA methylation of enhancers could play a role in the regulation of gene expression with age. However, we cannot rule out the reverse causality of ageassociated expression affecting methylation profiles or uncontrolled (hidden) variation resulting in the correlation between methylation and gene expression. Additionally, the majority of the age and gene expression associations (direct effects) remained significant after adjusting for $\mathrm{CPG}$ methylation, suggesting that regulators other than the measured CpG methylation likely contribute to the relationship between age and gene expression. Moreover, further investigations of other potential drivers for gene expression changes with age are warranted.

\section{Transcriptomic profiles associating with pulse pressure}

To see if age-related changes in gene expression may also reflect vascular age, we examined the relationships between age-associated gene expression profiles and a surrogate of vascular age, pulse pressure. Of the 2,704 genes associated with age $(\mathrm{FDR} \leq 0.001)$ in the 1,264 monocyte samples, 15 genes were also associated with pulse pressure $\left(\mathrm{FDR}_{\text {genome-wide }} \leq 0.01\right)$, after adjusting for age and appropriate biological and technical covariates (Additional file 2: Table S11). The gene most significantly associated with pulse pressure was PTGER2 (prostaglandin E receptor 2 (subtype EP2)), which had increasing expression associated with higher pulse pressure $\left(\mathrm{FDR}_{\text {genome-wide }}=3.15 \times 10^{-7}\right)$. Additionally, the increasing expression of $M C L 1$, a known inhibitor of autophagy [29] and one of the most significant associations we detected with age in monocytes, was also independently associated with higher pulse pressure.

\section{Transcriptomic profiles associated with age in $423 \mathrm{~T}$ cell and monocyte samples}

We carried out transcriptomic profiling of CD4+ T cell samples using microarrays (Illumina HumanHT-12 v4 Expression BeadChip) for a subset of the MESA samples with transcriptomic data in monocytes $(n=423)$, and detected 10,322 genes expressed in both $\mathrm{T}$ cell and monocyte samples (Figure 1B). A comparison of the effect of age on gene expression in $\mathrm{T}$ cells and monocytes is shown in Figure 5, which reveals 188 genes with expression significantly associated with age only in T cells, 383 genes associated with age only in monocyte samples, and 30 genes with age-associated expression in both the $\mathrm{T}$ cell and monocyte samples (FDR $<0.01$, Additional file 2: Table S12). The majority (93\%) of the genes detected 
Table 2 Age-associated methylation predicted to mediate the relationship between age and expression of oxidative phosphorylation, ribosome, and autophagy genes

\begin{tabular}{|c|c|c|c|c|c|c|c|c|c|c|c|c|}
\hline \multicolumn{4}{|c|}{ Age Methylation } & \multicolumn{3}{|c|}{ Methylation cis Gene expression } & \multicolumn{6}{|c|}{ Age $\sim$ Gene expression } \\
\hline \multirow[t]{2}{*}{ CpG ID } & \multirow[t]{2}{*}{ Chr } & \multirow[t]{2}{*}{ Cor } & \multirow[t]{2}{*}{ FDR } & \multirow[t]{2}{*}{ Gene } & \multirow[t]{2}{*}{ Cor } & \multirow[t]{2}{*}{ FDR } & \multicolumn{2}{|l|}{ Indirect } & \multicolumn{2}{|l|}{ Direct } & \multicolumn{2}{|l|}{ Total } \\
\hline & & & & & & & Beta (SE) & P-value & Beta (SE) & P-value & Beta (SE) & FDR \\
\hline \multicolumn{13}{|c|}{ Oxidative phosphorylation genes from gene ontology (GO:0006119): } \\
\hline $\operatorname{cg} 07388493$ & 1 & -0.4 & $2.4 \mathrm{E}-42$ & NDUFS5 & 0.22 & $1.1 \mathrm{E}-12$ & $-0.097(0.014)$ & 2.7E-12 & $-0.09(0.03)$ & $1.9 \mathrm{E}-03$ & $-0.19(0.03)$ & $1.2 \mathrm{E}-08$ \\
\hline cg24704287 & 19 & -0.17 & $1.6 \mathrm{E}-07$ & NDUFBT & 0.16 & $1.3 \mathrm{E}-05$ & $-0.037(0.009)$ & $1.8 \mathrm{E}-05$ & $-0.11(0.03)$ & $1.8 \mathrm{E}-05$ & $-0.15(0.03)$ & $9.4 \mathrm{E}-06$ \\
\hline cg09267188 & 8 & -0.14 & $2.1 \mathrm{E}-05$ & $U Q C R B$ & -0.13 & $1.1 \mathrm{E}-03$ & $0.028(0.007)$ & 3.7E-05 & $0.10(0.03)$ & $9.3 \mathrm{E}-05$ & $0.13(0.03)$ & $1.0 \mathrm{E}-05$ \\
\hline $\operatorname{cg} 27246938$ & 7 & -0.11 & $7.5 \mathrm{E}-04$ & NDUFB2 & -0.24 & $2.5 E-14$ & $0.030(0.007)$ & $6.1 \mathrm{E}-05$ & $-0.14(0.03)$ & 1.1E-07 & $-0.11(0.03)$ & $1.2 \mathrm{E}-\mathrm{O}$ \\
\hline cg09876992 & 22 & -0.32 & $3.3 \mathrm{E}-26$ & NDUFA6 & -0.13 & $8.5 \mathrm{E}-04$ & $-0.027(0.007)$ & $1.8 \mathrm{E}-04$ & $-0.17(0.03)$ & 7.2E-10 & $-0.20(0.03)$ & $6.9 \mathrm{E}-04$ \\
\hline \multicolumn{13}{|c|}{ Ribosome genes from gene ontology (GO:005840): } \\
\hline $\operatorname{cg} 17328880$ & 19 & -0.25 & $3.4 \mathrm{E}-16$ & MRPL34 & 0.29 & $4.0 \mathrm{E}-22$ & $-0.070(0.01)$ & $7.6 \mathrm{E}-12$ & $-0.07(0.03)$ & $4.8 \mathrm{E}-03$ & $-0.14(0.03)$ & $3.0 \mathrm{E}-06$ \\
\hline $\operatorname{cg} 04865726$ & 1 & -0.27 & $7.5 \mathrm{E}-19$ & MRPL20 & 0.24 & $1.2 \mathrm{E}-14$ & $-0.069(0.011)$ & 7.7E-11 & $-0.12(0.03)$ & $3.9 \mathrm{E}-06$ & $-0.19(0.03)$ & $9.2 \mathrm{E}-09$ \\
\hline cg08885076 & 2 & -0.2 & $8.1 \mathrm{E}-11$ & MRPL30 & 0.2 & $7.6 \mathrm{E}-10$ & $-0.042(0.008)$ & $6.9 \mathrm{E}-08$ & $-0.05(0.03)$ & $5.3 \mathrm{E}-02$ & $-0.09(0.03)$ & $1.1 \mathrm{E}-03$ \\
\hline $\operatorname{cg} 16399745$ & 12 & -0.29 & $6.4 \mathrm{E}-21$ & MRPL51 & -0.15 & $8.4 \mathrm{E}-05$ & $0.036(0.008)$ & $5.5 \mathrm{E}-06$ & $-0.15(0.03)$ & 1.1E-08 & $-0.12(0.03)$ & $6.6 \mathrm{E}-05$ \\
\hline $\operatorname{cg} 16604233$ & 6 & -0.26 & 3.0E-17 & RPS18 & -0.16 & $4.6 \mathrm{E}-06$ & $0.042(0.01)$ & $1.6 \mathrm{E}-05$ & $-0.16(0.03)$ & $8.1 \mathrm{E}-08$ & $-0.11(0.03)$ & $1.3 \mathrm{E}-04$ \\
\hline $\operatorname{cg} 16000022$ & 11 & -0.16 & $1.8 \mathrm{E}-06$ & MRPL21 & -0.15 & $4.9 \mathrm{E}-05$ & $0.028(0.007)$ & $2.8 \mathrm{E}-05$ & $0.10(0.03)$ & $1.3 \mathrm{E}-04$ & $0.13(0.03)$ & $6.9 \mathrm{E}-0$ \\
\hline 17614703 & 5 & 0.14 & $3.2 \mathrm{E}-05$ & CANX & -0.13 & $77 E_{0} 04$ & $0.028(0.007)$ & $2.9 \mathrm{E}-05$ & $0.10(0.03)$ & $8.2 \mathrm{E}-05$ & $0.13(0.03)$ & $3.2 \mathrm{E}-05$ \\
\hline cg15829665 & 6 & -0.12 & $5.5 \mathrm{E}-04$ & MRPL18 & -0.13 & $1.3 \mathrm{E}-03$ & $0.028(0.007)$ & $3.6 \mathrm{E}-05$ & $0.10(0.03)$ & 9.3E-05 & $0.13(0.03)$ & $4.6 \mathrm{E}-05$ \\
\hline cg21252105 & 9 & -0.18 & $2.8 \mathrm{E}-08$ & MRPL41 & 0.15 & $4.3 \mathrm{E}-05$ & $-0.035(0.009)$ & $7.8 \mathrm{E}-05$ & $-0.10(0.03)$ & $2.2 \mathrm{E}-04$ & $-0.13(0.03)$ & $2.7 \mathrm{E}-0$ \\
\hline $\operatorname{cg} 14663914$ & 19 & -0.11 & $1.1 \mathrm{E}-03$ & RPS15 & 0.17 & $1.7 \mathrm{E}-06$ & $-0.027(0.007)$ & $8.9 \mathrm{E}-05$ & $-0.17(0.03)$ & $1.5 \mathrm{E}-09$ & $-0.20(0.03)$ & $6.5 \mathrm{E}-06$ \\
\hline cg05017994 & 5 & 0.16 & $9.4 \mathrm{E}-07$ & MRPL36 & -0.15 & $2.3 \mathrm{E}-05$ & $-0.039(0.01)$ & $9.6 \mathrm{E}-05$ & $-0.14(0.03)$ & $3.2 \mathrm{E}-07$ & $-0.18(0.03)$ & $1.9 \mathrm{E}-0$ \\
\hline $\operatorname{cg} 23163653$ & 6 & -0.16 & $3.8 \mathrm{E}-07$ & $A B C F 1$ & -0.13 & $6.2 \mathrm{E}-04$ & $-0.027(0.007)$ & $1.0 \mathrm{E}-04$ & $-0.17(0.03)$ & $1.2 \mathrm{E}-09$ & $-0.20(0.03)$ & $1.3 \mathrm{E}-03$ \\
\hline $\operatorname{cg} 10700019$ & 8 & -0.11 & $9.8 \mathrm{E}-04$ & RPL8 & -0.28 & $5.2 \mathrm{E}-21$ & $0.033(0.008)$ & 1.1E-04 & $0.12(0.03)$ & 7.7E-06 & $0.15(0.03)$ & $1.0 \mathrm{E}-0$ \\
\hline cg27209993 & 6 & -0.14 & $2.7 \mathrm{E}-05$ & MRPS10 & 0.17 & $4.0 \mathrm{E}-07$ & $-0.023(0.006)$ & $1.3 \mathrm{E}-04$ & $-0.06(0.03)$ & 2.7E-02 & $-0.09(0.03)$ & $1.1 \mathrm{E}-\mathrm{C}$ \\
\hline cg00435173 & 17 & -0.2 & 3.7E-10 & RPL27 & 0.13 & $1.1 \mathrm{E}-03$ & $0.033(0.009)$ & $1.4 \mathrm{E}-04$ & $0.12(0.03)$ & $4.8 \mathrm{E}-06$ & $0.15(0.03)$ & $8.0 \mathrm{E}-\mathrm{C}$ \\
\hline cg00530414 & 16 & -0.21 & $8.3 E-12$ & RPS15A & -0.15 & $3.9 \mathrm{E}-05$ & $0.030(0.008)$ & $1.8 \mathrm{E}-04$ & $-0.13(0.03)$ & $5.0 \mathrm{E}-06$ & $-0.09(0.03)$ & $1.0 \mathrm{E}-03$ \\
\hline cg22803868 & 17 & -0.12 & $6.1 \mathrm{E}-04$ & NUFIP2 & -0.14 & $2.0 \mathrm{E}-04$ & $0.023(0.007)$ & 4.7E-04 & $0.11(0.03)$ & $8.8 \mathrm{E}-05$ & $0.13(0.03)$ & $7.1 \mathrm{E}-$ \\
\hline $\operatorname{cg} 13084677$ & 4 & 0.12 & $3.8 \mathrm{E}-04$ & $R P L 9$ & 0.19 & $3.5 \mathrm{E}-09$ & $0.017(0.006)$ & 4.6E-03 & $-0.12(0.03)$ & $1.8 \mathrm{E}-05$ & $-0.10(0.03)$ & 7.7E-04 \\
\hline \multicolumn{13}{|c|}{ Autophagy genes from Figure 3: } \\
\hline $\operatorname{cg} 24213719$ & 18 & 0.19 & $1.9 \mathrm{E}-09$ & $B C L 2$ & 0.13 & $7.2 \mathrm{E}-04$ & $0.028(0.007)$ & 3.3E-05 & $0.10(0.03)$ & $1.4 \mathrm{E}-04$ & $0.13(0.03)$ & $1.2 \mathrm{E}-03$ \\
\hline $\operatorname{cg} 11789534$ & 22 & -0.17 & $1.3 \mathrm{E}-07$ & $\operatorname{IL} 2 R B$ & 0.16 & 4.1E-06 & $-0.032(0.008)$ & 4.6E-05 & $0.09(0.02)$ & 4.2E-05 & $0.06(0.02)$ & $9.9 \mathrm{E}-04$ \\
\hline cg21826784 & 1 & -0.12 & $3.4 \mathrm{E}-04$ & FRAP1 & -0.13 & $9.5 \mathrm{E}-04$ & $-0.027(0.007)$ & $1.2 \mathrm{E}-04$ & $-0.17(0.03)$ & $2.2 \mathrm{E}-09$ & $-0.20(0.03)$ & $2.3 \mathrm{E}-0$ \\
\hline $\operatorname{cg} 22117188$ & 3 & -0.13 & $1.4 \mathrm{E}-04$ & PRKCD & -0.18 & $1.9 \mathrm{E}-07$ & $-0.027(0.007)$ & $1.5 \mathrm{E}-04$ & $-0.17(0.03)$ & $5.2 \mathrm{E}-10$ & $-0.20(0.03)$ & $8.6 \mathrm{E}-05$ \\
\hline cg18728264 & 11 & -0.19 & $1.9 \mathrm{E}-09$ & ILIORA & 0.14 & $3.9 \mathrm{E}-04$ & $-0.020(0.006)$ & 4.3E-04 & $0.21(0.03)$ & 1.7E-13 & $0.19(0.03)$ & $1.5 \mathrm{E}-08$ \\
\hline
\end{tabular}

CpGs whose degree of methylation significantly associated with age (FDR $\leq 0.001)$, cis-gene expression $( \pm 1 \mathrm{MB}$; FDR $\leq 0.001)$, and was predicted to mediate (indirect p-value $<0.05$ ) the total effect (total beta, SE, FDR) of age on gene expression. Results include only the most significant mediating CpG identified per gene for oxidative phosphorylation genes (from Gene Ontology pathway GO:0006119), ribosome genes (from GO:005840) and autophagy genes (from Figure 3), and are sorted first by pathway, then by the significance of the mediation effect (full mediation results are presented in Additional file 2: Table S10). The direct effects of age on gene expression not supported to be mediated by methylation are also shown (direct beta, SE, p-value). Analyses included 1,264 CD14+ monocyte samples; partial correlations (cor) were adjusted for sex, race, study site, residual contamination with non-targeted cells, and microarray chip effects.

with age-associated expression in the subset of 423 monocyte samples were also significantly (FDR $\leq$ 0.001 ) associated with age in the full sample of 1,264 monocytes, with similar effect directions observed for all genes (Additional file 1: Figure S7 and Additional file 2: Table S13).
Age-associated genes identified in the subset of 423 monocyte samples were enriched with ribonucleoprotein complex genes, similar to results from the expanded sample size of monocytes. After stratifying by the effect direction, the genes with expression negatively associated with age were enriched with ribonucleoprotein 


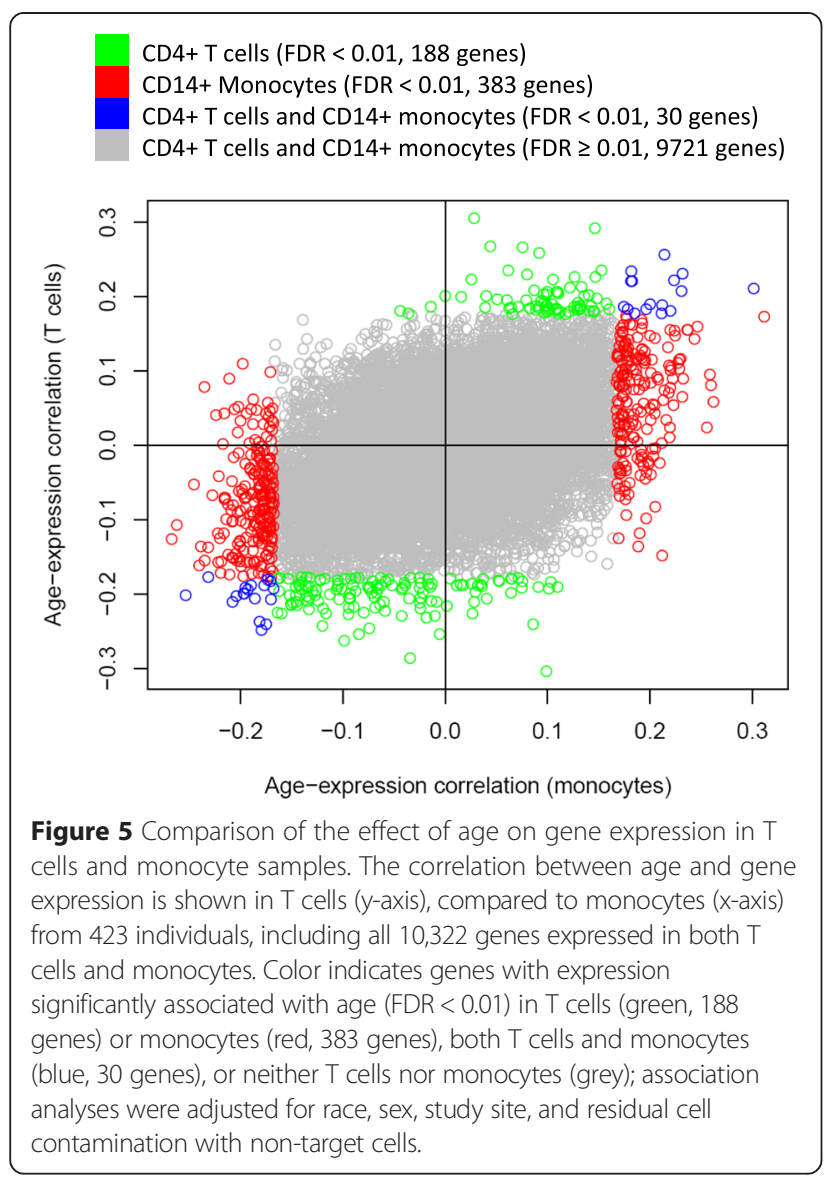

complex and mitochondrion genes, while genes with expression positively associated with age were enriched for cellular biosynthetic processes (Additional file 1: Table S14). The down-regulated mitochondria genes included ATP synthase complex genes (ATP5E, ATP5S, ATP5G1, ATP5I, ATP5G3) and electron transport chain genes (NDUFS5, TXNRD1, NDUFS3, CRYZL1) which are key genes for oxidative phosphorylation.

No pathways were significantly (FDR $<0.05)$ enriched among genes with age-associated expression in T cells; however, there was suggestive enrichment for the ribonucleoprotein complex among genes with expression negatively associated with age, and for the immune response pathway among genes with expression positively associated with age (Additional file 1: Table S14). Providing further evidence for a transcriptional decline of ribosomal protein synthesis genes with age in $\mathrm{T}$ cells, the majority $(62 \%)$ of the ribonucleoprotein complex genes with expression profiles negatively associated with age in monocytes were also negatively associated with age in $\mathrm{T}$ cells $(\mathrm{p}<0.05)$. However, the overall decline of oxidative phosphorylation gene expression with older age that was detected in monocytes was not detectable in T cells.

These results, from a large number of purified $\mathrm{T}$ cell and monocyte samples from the same individuals, identify only a small number of genes with transcriptomic profiles associated with aging in both cell types, supporting the idea that some age-related changes may be cell-type specific. However, the potential decline in protein synthesis machinery gene expression observed in both cell types, and previously reported in human blood leukocytes [10] and brain tissue [6], further support the hypothesis that some transcriptomic changes are conserved to varying degrees across cell types.

\section{Limitations of the study}

Several limitations of the study should be noted. Our investigation included adults aged 55 to 94 years; therefore, these results may not be applicable to younger populations. Also, our primary analysis used microarrays to measure gene expression rather than RNA sequencing, which may have missed low abundance genes. The cross-sectional nature of the investigation also limits inferences for the associations of gene expression with chronological age. Longitudinal analyses are necessary to confirm the effect of age on expression of identified genes and gene networks. We also acknowledge that the analyses of $\mathrm{CpG}$ methylation as a potential mediator of the effect of age on gene expression should be interpreted with caution since statistical mediation does not differentiate correlation from causation. Lastly, some of the age-associated transcriptional differences we observed may not reflect differences in protein levels or protein activity, although we have quantified protein levels using western blot for two of our transcriptional signals.

\section{Conclusions}

In this transcriptomic study of purified monocytes from a large, multi-ethnic and mixed gender population, older age appears to be associated with a transcriptomic decline in ribosomal protein synthesis machinery, oxidative phosphorylation, and autophagy pathways. The ability to detect a large number of biologically plausible gene expression changes support the use of CD14+ monocytes, a readily accessible cell population, as a model for further investigations of human aging, including the potential decline of autophagy and mitochondrial biogenesis with age. Our data also provides clues to the potential drivers of these transcriptomic changes with age, such as chromatin remodeler genes and DNA methylation. Further functional work is required to investigate the causes and consequences of these mRNA expression alterations with age.

Our sample size of purified $\mathrm{T}$ cells from a subset of the population is also the largest reported to date, which allowed sufficient power to detect age-sensitive genes, and provided suggestive evidence for transcriptomic alterations in ribosomal protein synthesis machinery and 
immune response pathways with age. The full list of age-genes identified from either CD4+ T cells or CD14+ monocytes harbors many strong candidate genes for future studies of the aging process. In designing such experimental studies one may want to consider that there may be tissue- or cell-specific changes with age, although some patterns of aging are likely similar between different human tissues.

\section{Methods}

\section{Study population}

The Multi-Ethnic Study of Atherosclerosis (MESA) was designed to investigate the prevalence, correlates, and progression of subclinical cardiovascular disease in a population cohort of 6,814 participants. Since its inception in 2000, five clinic visits collected extensive clinical, socio-demographic, lifestyle, behavior, laboratory, nutrition, and medication data [60]. The present analysis is based on analyses of purified monocyte and T cell samples from the April 2010 - February 2012 examination (Exam 5) of 1,264 randomly selected MESA participants (55 - 94 years old, Caucasian (47\%), African American (21\%) and Hispanic (32\%), female (51\%)) from four MESA field centers (Baltimore, MD; Forsyth County, NC; New York, NY; and St. Paul, MN). The study protocol was approved by the Institutional Review Boards at Johns Hopkins Medical Institutions, Wake Forest University Health Sciences, Columbia University Medical Center, and the University of Minnesota. All participants signed informed consent.

\section{Purification of CD14+ Monocytes and CD4+ T cells}

Centralized training of technicians, standardized protocols, and extensive quality control $(\mathrm{QC})$ measures were implemented for collection, on-site processing, and shipment of MESA specimens, and routine calibration of equipment was performed. Blood was initially collected in sodium heparin-containing Vacutainer CPTTM cell separation tubes (Becton Dickinson, Rutherford, NJ) to separate peripheral blood mononuclear cells from other elements within two hours from blood draw. Subsequently, monocytes and $\mathrm{T}$ cells were isolated with antiCD14 and anti-CD4 monoclonal antibody coated magnetic beads, respectively, using autoMACS automated magnetic separation unit (Miltenyi Biotec, Bergisch Gladbach, Germany). Initially flow cytometry analysis of 18 specimens was performed, including samples from all four MESA field centers, which were found to be consistently $>90 \%$ pure.

\section{DNA/RNA extraction}

DNA and RNA were isolated from samples simultaneously using the AllPrep DNA/RNA Mini Kit (Qiagen, Inc., Hilden, Germany). DNA and RNA QC metrics included optical density (OD) measurements, using a NanoDrop spectrophotometer and evaluation of the integrity of $18 \mathrm{~s}$ and $28 \mathrm{~s}$ ribosomal RNA using the Agilent 2100 Bioanalyzer with RNA 6000 Nano chips (Agilent Technology, Inc., Santa Clara, CA) following manufacturer's instructions. RNA with RIN (RNA Integrity) scores $>9.0$ was used for global expression microarrays. The median of RIN for our 1,264 samples was 9.9.

\section{Global expression quantification}

The Illumina HumanHT-12 v4 Expression BeadChip and Illumina Bead Array Reader were used to perform the genome-wide expression analysis, following the Illumina expression protocol. The Illumina TotalPrep-96 RNA Amplification Kit (Ambion/Applied Biosystems, Darmstadt, Germany) was used for reverse transcription, and amplification with $500 \mathrm{ng}$ of input total RNA (at 11ul). $700 \mathrm{ng}$ of biotinylated cRNA was hybridized to a BeadChip at $58^{\circ} \mathrm{C}$ for $16-17$ hours. To avoid potential biases due to batch, chip, and position effects, a stratified random sampling technique was used to assign individual samples (including five common control samples for the first 480 samples) to specific BeadChips (12 samples/ chip) and chip position.

\section{Epigenome-wide methylation quantification}

The Illumina HumanMethylation450 BeadChip and HiScan reader were used to perform the epigenome-wide methylation analysis. The EZ-96 DNA Methylation "' Kit (Zymo Research, Orange, CA) was used for bisulfate conversation with $1 \mu \mathrm{g}$ of input DNA (at $45 \mu \mathrm{l}$ ). $4 \mu \mathrm{l}$ of bisulfiteconverted DNA were used for DNA methylation assays, following the Illumina Infinium HD Methylation protocol. This consisted of a whole genome amplification step followed by enzymatic end-point fragmentation, precipitation, and resuspension. The resuspended samples were hybridized on HumanMethylation 450 BeadChips at $48^{\circ} \mathrm{C}$ for $16 \mathrm{~h}$. The individual samples were assigned to the BeadChips and to chip position using the same sampling scheme as that for the expression BeadChips.

\section{Quality control and Pre-processing of microarray data}

Data pre-processing and quality control $(\mathrm{QC})$ analyses were performed in $R$ (http://www.r-project.org/) using Bioconductor (http://www.bioconductor.org/) packages. For expression data, data corrected for local background were obtained from Illumina's proprietary software GenomeStudio. QC analyses and bead type summarization (average bead signal for each type after outlier removal) were performed using the beadarray package [61]. Detection P-values were computed using the negative controls on the array. The neqc function of the limma [62] package was used to perform a normal-exponential convolution model analysis to estimate non-negative signal, quantile normalization using 
all probes (gene and control, detected and not detected) and samples, addition of a recommended (small) offset, $\log _{2}$ transformation, and elimination of control probe data from the normalized expression matrix. Multidimensional scaling plots showed the five common control samples were highly clustered together and identified three outlier samples, which were excluded subsequently. For both monocyte and T cell assays, we included $2 \%$ blind duplicates. Correlations among technical replicates exceeded 0.997.

The Illumina HumanHT-12 v4 Expression BeadChip included $>47,000$ probes for $>30,000$ genes (with unique Entrez gene IDs). Statistical analyses excluded probes with non-detectable expression in $\geq 90 \%$ of MESA samples (using a detection p-value cut-off of 0.0001), probes overlapping repetitive elements or regions, probes with low variance across the samples ( $<10$ th percentile), or probes targeting putative and/or not well-characterized genes, i.e. gene names starting with KIAA, FLJ, HS, MGC, or LOC.

Bead-level methylation data were summarized in GenomeStudio. Because the Illumina HumanMethylation450 BeadChip technology employs a two-channel system and uses both Infinium I and II assays, normalization was performed in several steps using the lumi package [63]. We first adjusted for color bias using "smooth quantile normalization". Next, the data were background adjusted by subtracting the median intensity value of the negative control probes. Lastly, data were normalized across all samples by standard quantile normalization applied to the bead-type intensities and combined across Infinium I and II assays and both colors. QC measures included checks for sex and race/ ethnicity mismatches, and outlier identification by multidimensional scaling plots. The final methylation value for each methylation probe was computed as the M-value, essentially the log ratio of the methylated to the unmethylated intensity [64]. The M-value is well suited for high-level analyses and can be transformed into the beta-value, an estimate of the percent methylation of an individual $\mathrm{CpG}$ site that ranges from 0 to 1 (thus $\mathrm{M}$ is logit(beta-value)).

The Illumina HumanMethylation450 BeadChip included probes for $>485,000$ CpGs. Statistical analyses excluded CpGs with: "detected" methylation levels in $<90 \%$ of MESA samples using a detection p-value cut-off of 0.05 , existence of any SNPs within 10 base pairs of the targeted $\mathrm{CpG}$, or overlap with a repetitive element or region.

Pre-processing with global normalization removed large position and chip effects across all probes; however, probe-specific chip effects were found for some CpGs and gene transcripts, while probe-specific position effects existed for some CpGs but were ignorable for all gene transcripts. These probe-specific effects were included as covariates in all subsequent analyses.

\section{Pulse pressure measures}

Blood pressure was measured 3 times at 2-minute intervals using an automated oscillometric device (Dinamap Monitor Pro 100, GE Healthcare, Milwaukee, WI) after participants had rested for five minutes in the seated position (MESA Exam 5). Appropriately sized cuffs were used for blood pressure assessment. Blood pressure was defined as the average of the second and third readings. The average systolic and diastolic blood pressure values were used to calculate pulse pressure, which was defined as systolic minus diastolic blood pressure.

\section{Association analyses}

The overall goal of the association analysis was to identify associations, at the genome-wide level, between age and gene expression, age and $\mathrm{CpG}$ methylation, and transcript expression and $\mathrm{CpG}$ methylation. Association analyses were performed using the linear model $(\mathrm{lm})$ function of the Stats package and the stepAIC function of the MASS package in $R$. To identify gene transcripts or methylation sites associated with age, we fit separate linear regression models with age as a predictor of transcript expression or the $\mathrm{M}$-value for each gene transcript or CpG site, respectively. Covariates were sex, race/ethnicity, study site, expression/methylation chip, methylation position (for age-CpG methylation analyses only), and residual sample contamination with non-targeted cells (e.g. non-monocytes, see below). To identify methylation sites associated with gene expression in cis, we fit separate linear regression models with the $\mathrm{M}$-value for each CpG site (adjusted for methylation chip and position effects) as a predictor of transcript expression for any autosomal gene within $1 \mathrm{Mb}$ of the $\mathrm{CpG}$ in question. Covariates were age, sex, and race/ethnicity, study site, expression chip, and residual sample contamination with non-targeted cells. Sex- and ethnicity-stratified analyses were performed as an internal validation and check of generalizability. To look for potential population stratification, we used EIGENSTRAT [65] to compute principal components (PCs) for each race, based on Affymetrix 6.0 array genotype data [66], and examined the association between the first five PCs and gene expression, as well as CpG methylation, in race stratified analyses. Less than $1 \%$ of expression transcripts and CpG methylation sites in monocytes were associated with PCs in the Caucasian and African American populations (FDR $<0.05)$. However, $14.7 \%$ of gene expression transcripts and $3.1 \%$ of methylation sites in the Hispanic population were associated with the first two PCs $(\mathrm{FDR}<0.05)$; therefore, analyses in the Hispanic population were adjusted for the first two PCs. P-values were adjusted for multiple 
testing using the q-value FDR method [67]. The reported FDR was calculated at the genome-wide level for all genes, CpGs, or cis-gene/CpGs that were tested.

Association analyses for individual gene transcripts and pulse pressure were performed using the linear model $(l m)$ function in R. We fit separate linear regression models with transcript expression as a predictor of pulse pressure. Covariates included age, sex, race/ethnicity, study site, expression/methylation chip, methylation position (for age-CpG methylation analyses only), and residual sample contamination.

To estimate residual sample contamination for monocyte and $\mathrm{T}$ cell data analysis, we generated separate enrichment scores for neutrophils, B cells, T cells, monocytes, and natural killer cells. We implemented a Gene Set Enrichment Analysis [68] to calculate the enrichment scores using the gene signature of each blood cell type in the ranked list of expression values for each MESA sample. The cell type-specific signature genes were selected from previously defined lists [69] and passed the following additional filters: at least four-fold more highly expressed in the targeted cell type than in other cell populations and low expression levels in the targeted cells.

\section{Functional annotation analysis}

DAVID Bioinformatics Resources 6.7 was used to examine the enrichment (FDR < 0.05) of GO (Gene Ontology) pathways among gene lists, relative to all genes expressed and passing QC) [22,23]. Experimentally determined protein-protein interactions listed in STRING (Search Tool for the Retrieval of Interacting Genes/ Proteins v9.05 and v9.10) [35] were used to create networks of biological connections. Cytoscape [70] was used to visualize protein-protein interactions reported by STRING.

\section{Weighted gene Co-expression network analyses}

For gene network analysis we pre-selected ageassociated genes at a less stringent FDR level of 0.01, resulting in a subset of 4,129 genes. To cluster the subset of 4,129 genes into network modules of highly correlated transcripts, we applied the Weighted Gene Co-Expression Network Analysis as implemented in the $\mathrm{R}$ package WGCNA [24]. We used this method to first construct a weighted network based on the pairwise correlations among all transcripts considered, using soft thresholding with parameter values chosen to produce approximately a scale-free topology. Then, using the topological overlap measure to estimate the network interconnectedness, the transcripts were hierarchically clustered. We used the default parameters of WGCNA, except for changing the correlation type from Pearson to biweight midcorrelation (which is more robust to outliers) and set the minimum size for module detection from 20 to 10 . For each module, we obtained the eigengene (the first eigenvector of the within-module expression correlation matrix, or the first right-singular vector of the standardized within-module expression matrix).

\section{Stability analysis and consensus modules}

Unfortunately, the module structure identified by WGCNA tends to be rather unstable, even when the sample size is relatively large (in the hundreds). Stability of the module structure can be assessed by repeatedly making relatively small, random changes to the data and re-running the analysis, and then assessing the agreement between the resulting structures. One way of making such changes to the data is by sampling random subsets of the data ("sub-sampling") which contain most but not all of the samples. We performed sub-sampling by obtaining a random sample of $80 \%$ of the observations (MESA participants), performing WGCNA on this data subset (with module detection) and repeating this process 200 times. Each of the 200 module assignment was represented by an unsigned network in which all transcripts assigned to the same module were connected by an edge. The Jaccard index [71] was used to evaluate the similarity between any two networks and is equal to the number of edges shared by two networks divided by the total number of edges in present in either network. Hence, the Jaccard index ranges from 0 to 1 , with larger values indicating higher similarity between two networks The values of the Jaccard index for the network constructed from the original data and any network obtained from a sub-sampled data set were low with mean value (across 200 replicates) in the range $0.25-0.30$. To increase the stability of the module assignment, we calculated a consensus network composed of those edges which were present in at least $70 \%$ of the 200 networks constructed from the sub-sampled data sets (no minimum size for consensus network modules). We then compared several consensus networks, each based on 200 subsamples, resulting in Jaccard index values very near 0.90 and indicating much higher stability between consensus networks compared with the (in)stability of networks from individual datasets.

\section{mRNA quantification using RNA seq}

Expression levels accessed by microarray were compared to results from RNA-sequencing in the subset of the monocyte samples $(n=373)$, indicating excellent reproducibility of microarray data (correlations ranged from 0.45-0.86, median: 0.76). Detailed information describing mRNA quantification is provided in the Additional file 1. 


\section{MCL1 and MRPS12 protein extraction and western blotting}

Following DNA/RNA extraction, protein pellet was precipitated from RLT Plus buffer (Qiagen, Inc., Hilden, Germany) with acetone per manufacturer instructions. Pellet was resuspended in $100 \mu \mathrm{l}$ modified $4 \times$ Laemmli buffer [72] (4\% SDS, $250 \mathrm{mM}$ Tris $\mathrm{HCl}$, no glycerol, no bromophenol blue, no $\beta$-mercaptoethanol) mixed 1:1 with 8 M Urea [73], with SigmaFAST Protease Inhibitor Cocktail Tablet (Sigma-Aldrich, St. Louis, MO). Samples were warmed to $55^{\circ} \mathrm{C}$, and sonicated $4 \times 30$ seconds in a Bioruptor (Diagenode, Denville, NJ). Protein concentration was determined using bicinchoninic acid microplate assay (Thermo Scientific, Rockford, IL). Samples were mixed 1:4 with 5x Loading Buffer Supplement (50\% glycerol, $0.02 \%$ bromphenol blue, $12.5 \% \beta$-mercaptoethanol), separated by SDS-PAGE on NuPage Novex 4-12\% BisTris Midi gels (Life Technologies, Grand Island, NY), and transferred to Immobilon Fl (Millipore, Billerica, MA) PVDF membranes. Blots were blocked in non-fat dry milk and incubated with antibodies to Mcl1 (Santa Cruz Biotechnology, Santa Cruz, CA) (clone S-19, 1:500 dilution), MRPS12 (Proteintech Group, Chicago, Il) (rabbit polyclonal, catalog \#15225-1-AP, 1:333 dilution), and GAPDH (Ablabs, Vancouver, British Columbia) (clone ga1r, 1:3000 dilution) overnight at $4^{\circ} \mathrm{C}$. Secondary detection was performed using IRDye 680 and 800 secondary antibodies (LI-COR, Lincoln, NE), and imaged on an Odyssey Classic scanner (LI-COR, Lincoln, NE).

Mcl-1 protein quantification: $\mathrm{Mcl}-1$ often appears as a doublet or triplet in western blot analysis, in agreement with our own observations. These multiple bands are thought to occur for a variety of reasons, including: an alternative initiation site [74], alternative RNA splicing [75], serine/threonine phosphorylation [76,77], and perhaps most notably, and proteolytic cleavage of the Nterminus $[74,78,79]$. The production, stability, and turnover of Mcl-1 variants is diverse, and thus we chose to focus our quantitation on the dominant, high molecular weight species $(40 \mathrm{kDa})$, which likely corresponds to the full length Mcl-1 protein. GAPDH was used as a loading control because our gene expression analysis showed it has low variance and no association with age (FDR = 0.32 ). Individual protein band quantification was performed using Image Studio software (LI-COR, Lincoln, $\mathrm{NE}$ ). Target protein content was corrected for the content of GAPDH in samples.

\section{Mediation analysis}

We performed mediation analysis to investigate the hypothesis that age may have an effect on gene expression mediated through methylation alteration. We used Structural Equation Modeling (SEM) with bootstrapping as implemented in the $\mathrm{R}$ package lavaan [80] to estimate direct and indirect effects (mediated through DNA methylation) of age on gene expression.

\section{Availability of supporting data}

Microarray data presented in this manuscript has been deposited in the NCBI Gene Expression Omnibus (GEO) repository and is accessible through GEO Series accession number GSE56047. Other supporting data are included in Additional file 1 and Additional file 2 .

\section{Additional files}

\begin{abstract}
Additional file 1: Figure S1. Age-associations with the monocyte transcriptome (page 3). Figure S2. Correlation between co-expression network modules (page 4). Figure S3. Scatterplot of gene expression and age for genes in the 'black' co-expression network module (page 5). Figure S4. Correlation between MCL1 expression measured by microarray and RNA-sequencing (page 6). Figure S5. MCL1 expression measured using Western Blot (page 7). Figure S6. MRPS12 expression measured using Western Blot (page 8). Figure S7. Comparison of the effect of age on gene expression in 1,264 monocyte samples compared to results from a subset of 423 samples (page 9). Table S1. Population characteristics (page 10). Table S3. Gene set enrichment analysis for age-associated genes in monocytes from 1,264 MESA participants (page 11). Table S4. Co-expression network modules associated with age (page 12). Table S14. Gene set enrichment analysis for age-associated genes in CD4+ T cells and CD14+ monocytes from 423 MESA participants (page 13). Supplementary Methods: mRNA quantification using RNA seq (page 14-15). Supplementary References (page 16)

Additional file 2: Table S2. Association between age and expression of 4,502 age-associated genes (FDR $\leq 0.01$ ) in 1,264 monocyte samples (Tab 1). Table S5. Association between age and expression of 54 oxidative phosphorylation genes (Tab 2). Table S6. Association between age and expression of 204 ribosomal genes (Tab 3). Table S7 Association between age and expression of 51 mitochondrial ribosome genes (Tab 4). Table S8. Enrichment of transcription factor binding sites (TFBS) among 'turquoise' module genes (Tab 5). Table S9. Regulators of transcription assigned to the 'turquoise' module negatively correlated with 'blue' module expression (Tab 6). Table S10. Age-associated genes harboring cismethylation sites associated with age and predicted to mediate the effect of age on cis-gene expression (Tab 7). Table S11. Gene expression associated with age (FDR $\leq 0.01)$ and a pulse pressure (FDR $\leq 0.01)($ Tab 8).

Table S12. Gene expression associated with age (FDR $<0.01)$ in CD4+ T cells or CD14+ monocytes from 423 individuals (Tab 9). Table S13. Comparison of age associations with gene expression in 423 CD14+ monocyte samples and in the expanded CD14+ monocyte sample size $(n=1264)($ Tab 10).
\end{abstract}

\section{Abbreviations}

FDR: False Discovery Rate; CpG: Cytosine-phosphate-guanine dinucleotide MESA: The Multi-Ethnic Study of Atherosclerosis; WGCNA: Weighted gene co-expression network analysis; Eigengene: The first eigenvector of the within-module expression correlation matrix, or the first right-singular vector of the standardized within-module expression matrix; GO: Gene Ontology; MCL1: Myeloid Cell Leukemia sequence 1; BCl-2: B-cell CLL/lymphoma 2; TSC22D3: TSC22 domain family, member 3; CEBPD: CCAAT/enhancer binding protein, delta; IL: Interleukin (IL-2, IL-6, IL10RA); STAT: Signal transducer and activator of transcription; ATG: Autophagy related (ATG3, ATG5, ATG7); MTOR: Mechanistic target of rapamycin; PI3K/Akt: Phosphatidylinositol 3 -kinase(PI3K)/Akt; JAK: Janus kinase; PDPK1: 3-phosphoinositide dependent protein kinase-1; IL2RB: Interleukin 2 receptor, beta; AMPK: AMP-activated kinase; ATP: Adenosine triphosphate; PRKAG1: Protein kinase, AMP-activated, gamma 1 non-catalytic subunit; BECN1: Beclin-1, autophagy related; ULK1: Unc-51-like kinase 1; HMGB1: High mobility group box 1; CASP: Caspase, apoptosis-related cysteine peptidase (CASP2, CASP8); FOXO3: Forkhead box O3; OXPHOS: Oxidative phosphorylation; 
MRP: Mitochondrial ribosome protein (MRPL30, MRPS12); PGC-1a: Peroxisome proliferator-activated receptor gamma, coactivator 1 alpha; TFB2M: Transcription factor B2, mitochondrial; TFAM: Transcription factor A, mitochondrial; MTERF: Mitochondrial transcription termination factor; NRF-2: GA binding protein transcription factor, alpha subunit $60 \mathrm{kDa}$; POLRMT: Polymerase (RNA) mitochondrial (DNA directed); TFBS: Transcription factor binding sites; FOXO4: Forkhead box O4; YY1: YY1 transcription factor; NFKB1: Nuclear factor of kappa light polypeptide gene enhancer in B-cells 1; AHR: Aryl hydrocarbon receptor; SWI/SNF genes: SWItch/Sucrose NonFermentable (ARID1A, SMARCA4, SMARCA2, SMARCC2); RPS6KA2: Ribosomal protein 56 kinase, 90 kDa, polypeptide 2; NUFIP2: Nuclear fragile X mental retardation protein interacting protein 2; NDUF: NADH dehydrogenase (NDUFB7, NDUFA6, NDUFAF1); MAP1LC3A: Microtubule-associated protein 1 light chain 3 alpha.

\section{Competing interests}

The authors declare that they have no competing interests.

\section{Authors' contributions}

YL oversaw the study, carried out experiments, researched the data, and wrote the manuscript. LMR, JD, and JRT carried out experiments, researched the data, and wrote the manuscript. IH, CEM, DH, RPT, carried out experiments, researched the data, and helped write the manuscript. GB, WP, SS, DRJ, MVT, DC, RGB, CJ, TFL, KL, NS, and AdIF carried out experiments and researched the data. SBK, DS, JD, KMD, TCR, and CG researched the data. All authors read and approved the final manuscript.

\section{Acknowledgements}

This research was supported by contracts N01-HC-from the National Heart, Lung and Blood Institute, by grants UL1-RR-024156 and UL1-RR-025005 from the National Center for Research Resources, and T32AG033534 from the National Institute of Aging. The MESA Epigenomics \& Transcriptomics Study was funded by NHLBI grant R01HL101250 to Wake Forest University Health Sciences. The authors thank the other investigators, the staff, and the participants of the MESA study for their valuable contributions. A full list of participating MESA investigators and institutions can be found at http://www.mesa-nhlbi.org.

\section{Author details \\ 'Department of Epidemiology and Prevention, Division of Public Health Sciences, Wake Forest School of Medicine, Winston-Salem, North Carolina 27157, USA. ${ }^{2}$ Department of Internal Medicine, Wake Forest School of Medicine, Winston-Salem, North Carolina 27157, USA. ${ }^{3}$ Department of Gerontology and Geriatric Medicine, J. Paul Sticht Center on Aging, Wake Forest School of Medicine, Winston-Salem, North Carolina 27157, USA. ${ }^{4}$ Department of Biostatistical Sciences, Division of Public Health Sciences, Wake Forest School of Medicine, Winston-Salem, North Carolina 27157, USA. ${ }^{5}$ CRS4 Bioinformatica, Pula 09010, Italy. ${ }^{6} \mathrm{FBN}$, Leibniz Institute for Farm Animal Biology, Genetics and Biometry, Mecklenburg-Vorpommern, Germany. ${ }^{7}$ Departments of Medicine and Epidemiology, Cardiovascular Health Research Unit, University of Washington, Seattle, Washington 98115 , USA. ${ }^{8}$ Departments of Medicine and Epidemiology, Columbia University, New York, New York 10032, USA. 'Department of Pathology, Wake Forest School of Medicine, Winston-Salem, North Carolina 27157, USA. ${ }^{10}$ Department of Pathology, Johns Hopkins University, Baltimore, Maryland 21205, USA. ${ }^{11}$ Division of Biostatistics, Washington University School of Medicine, St. Louis, Missouri 63110, USA. ${ }^{12}$ New York Academy of Medicine, New York, New York 10029, USA. ${ }^{13}$ Division of Epidemiology and Community Health, School of Public Health, University of Minnesota, Minneapolis, Minnesota 55454, USA. ${ }^{14}$ Virginia Bioinformatics Institute, Virginia Tech, Blacksburg, Virginia 24061, USA. ${ }^{15}$ Department of Molecular Medicine, Wake Forest School of Medicine, Winston-Salem, North Carolina 27157, USA. \\ ${ }^{16}$ Department of Pathology, University of Vermont, Colchester, Vermont 05446, USA.}

\section{Received: 4 December 2014 Accepted: 10 April 2015}

\section{Published online: 22 April 2015}

\section{References}

1. Cao JN, Gollapudi S, Sharman EH, Jia Z, Gupta S. Age-related alterations of gene expression patterns in human CD8+ T cells. Aging Cell. 2010;9:19-31.
2. Remondini D, Salvioli S, Francesconi M, Pierini M, Mazzatti DJ, Powell JR, et al. Complex patterns of gene expression in human $T$ cells during in vivo aging. Mol Biosyst. 2010;6:1983-92.

3. Bektas A, Zhang Y, Lehmann E, Wood III WH, Becker KG, Madara K, et al. Ageassociated changes in basal NF-kappaB function in human CD4+ T lymphocytes via dysregulation of PI3 kinase. Aging (Albany NY). 2014;6:957-74.

4. Nakamura S, Kawai K, Takeshita Y, Honda M, Takamura T, Kaneko S, et al. Identification of blood biomarkers of aging by transcript profiling of whole blood. Biochem Biophys Res Commun. 2012;418:313-8.

5. Marttila S, Jylhava J, Nevalainen T, Nykter M, Jylha M, Hervonen A, et al. Transcriptional analysis reveals gender-specific changes in the aging of the human immune system. PLoS One. 2013;8:e66229.

6. Kumar A, Gibbs JR, Beilina A, Dillman A, Kumaran R, Trabzuni D, et al. Age-associated changes in gene expression in human brain and isolated neurons. Neurobiol Aging. 2013;34:1199-209.

7. Gheorghe M, Snoeck M, Emmerich M, Back T, Goeman JJ, Raz V. Major aging-associated RNA expressions change at two distinct age-positions. BMC Genomics. 2014;15:132.

8. Glass D, Vinuela A, Davies MN, Ramasamy A, Parts L, Knowles D, et al. Gene expression changes with age in skin, adipose tissue, blood and brain. Genome Biol. 2013;14:R75.

9. Zahn JM, Sonu R, Vogel H, Crane E, Mazan-Mamczarz K, Rabkin R, et al. Transcriptional profiling of aging in human muscle reveals a common aging signature. PLoS Genet. 2006;2:e115.

10. Harries LW, Hernandez D, Henley W, Wood AR, Holly AC, Bradley-Smith RM, et al. Human aging is characterized by focused changes in gene expression and deregulation of alternative splicing. Aging Cell. 2011;10:868-78.

11. Goronzy JJ, Weyand CM. Understanding immunosenescence to improve responses to vaccines. Nat Immunol. 2013;14:428-36.

12. De MM, Modesti M, Ginaldi L. Phenotypic and functional changes of circulating monocytes and polymorphonuclear leucocytes from elderly persons. Immunol Cell Biol. 2004;82:415-20.

13. Shi C, Pamer EG. Monocyte recruitment during infection and inflammation. Nat Rev Immunol. 2011;11:762-74.

14. Liu Y, Ding J, Reynolds LM, Lohman K, Register TC, de la Fuente A, et al. Methylomics of gene expression in human monocytes. Hum Mol Genet 2013;22:5065-74

15. Rakyan VK, Down TA, Maslau S, Andrew T, Yang TP, Beyan H, et al. Human aging-associated DNA hypermethylation occurs preferentially at bivalent chromatin domains. Genome Res. 2010;20:434-9.

16. Hernandez DG, Nalls MA, Gibbs JR, Arepalli S, van der Brug M, Chong S, et al. Distinct DNA methylation changes highly correlated with chronological age in the human brain. Hum Mol Genet. 2011;20:1164-72.

17. Alisch RS, Barwick BG, Chopra P, Myrick LK, Satten GA, Conneely KN, et al. Age-associated DNA methylation in pediatric populations. Genome Res. 2012;22:623-32.

18. Hannum G, Guinney J, Zhao L, Zhang L, Hughes G, Sadda S, et al. Genome-wide methylation profiles reveal quantitative views of human aging rates. Mol Cell. 2013;49:359-67.

19. Ong ML, Holbrook JD: Novel region discovery method for Infinium $450 \mathrm{~K}$ DNA methylation data reveals changes associated with aging in muscle and neuronal pathways. Aging Cell 2013.

20. Day K, Waite L, Thalacker-Mercer A, West A, Bamman M, Brooks J, et al. Differential DNA methylation with age displays both common and dynamic features across human tissues that are influenced by CpG landscape. Genome Biol. 2013;14:R102.

21. Reynolds LM, Taylor JR, Ding J, Lohman K, Johnson C, Siscovick D, et al. Age-related variations in the methylome associated with gene expression in human monocytes and T cells. Nat Commun. 2014;5:5366.

22. Dennis Jr G, Sherman BT, Hosack DA, Yang J, Gao W, Lane HC, et al. DAVID: database for annotation, visualization, and integrated discovery. Genome Biol. 2003;4:3.

23. da Huang W, Sherman BT, Lempicki RA. Systematic and integrative analysis of large gene lists using DAVID bioinformatics resources. Nat Protoc. 2009;4:44-57.

24. Langfelder $\mathrm{P}$, Horvath S. WGCNA: an R package for weighted correlation network analysis. BMC Bioinformatics. 2008;9:559.

25. He C, Klionsky DJ. Regulation mechanisms and signaling pathways of autophagy. Annu Rev Genet. 2009;43:67-93.

26. Rubinsztein DC, Marino G, Kroemer G. Autophagy and aging. Cell. 2011;146:682-95. 
27. Pattingre S, Tassa A, Qu X, Garuti R, Liang XH, Mizushima N, et al. Bcl-2 antiapoptotic proteins inhibit Beclin 1-dependent autophagy. Cell. 2005; 122:927-39.

28. Garcia-Saez AJ. The secrets of the Bcl-2 family. Cell Death Differ. 2012;19:1733-40.

29. Germain M, Nguyen AP, Le Grand JN, Arbour N, Vanderluit JL, Park DS, et al. $\mathrm{MCL}-1$ is a stress sensor that regulates autophagy in a developmentally regulated manner. EMBO J. 2011;30:395-407.

30. Asselin-Labat ML, David M, Biola-Vidamment A, Lecoeuche D, Zennaro MC, Bertoglio J, et al. GILZ, a new target for the transcription factor FoxO3, protects T lymphocytes from interleukin-2 withdrawal-induced apoptosis. Blood. 2004;104:215-23.

31. Moore F, Santin I, Nogueira TC, Gurzov EN, Marselli L, Marchetti P, et al. The transcription factor C/EBP delta has anti-apoptotic and antiinflammatory roles in pancreatic beta cells. PLoS One. 2012;7:e31062.

32. Zhang Y, Sif S, DeWille J. The mouse C/EBPdelta gene promoter is regulated by STAT3 and Sp1 transcriptional activators, chromatin remodeling and c-Myc repression. J Cell Biochem. 2007;102:1256-70.

33. Cantwell CA, Sterneck E, Johnson PF. Interleukin-6-specific activation of the C/EBPdelta gene in hepatocytes is mediated by Stat3 and Sp1. Mol Cell Biol. 1998;18:2108-17.

34. Thomas LW, Lam C, Edwards SW. Mcl-1; the molecular regulation of protein function. FEBS Lett. 2010;584:2981-9.

35. Szklarczyk D, Franceschini A, Kuhn M, Simonovic M, Roth A, Minguez $P$, et al. The STRING database in 2011: functional interaction networks of proteins, globally integrated and scored. Nucleic Acids Res. 2011;39:D561-8.

36. Salminen A, Kaarniranta K, Kauppinen A. Beclin 1 interactome controls the crosstalk between apoptosis, autophagy and inflammasome activation: Impact on the aging process. Ageing Res Rev. 2012;12:520-34.

37. Egan DF, Shackelford DB, Mihaylova MM, Gelino S, Kohnz RA, Mair W, et al. Phosphorylation of ULK1 (hATG1) by AMP-activated protein kinase connects energy sensing to mitophagy. Science. 2011;331:456-61.

38. Xiao B, Sanders MJ, Underwood E, Heath R, Mayer FV, Carmena D, et al. Structure of mammalian AMPK and its regulation by ADP. Nature. 2011;472:230-3.

39. Tang D, Kang R, Livesey KM, Cheh CW, Farkas A, Loughran P, et al. Endogenous HMGB1 regulates autophagy. J Cell Biol. 2010;190:881-92.

40. Bergamini E, Cavallini G, Donati A, Gori Z. The role of autophagy in aging: its essential part in the anti-aging mechanism of caloric restriction. Ann N Y Acad Sci. 2007;1114:69-78.

41. Cuervo AM, Bergamini E, Brunk UT, Droge W, Ffrench M, Terman A. Autophagy and aging: the importance of maintaining "clean" cells. Autophagy. 2005;1:131-40.

42. Terman A. The effect of age on formation and elimination of autophagic vacuoles in mouse hepatocytes. Gerontology. 1995;41 Suppl 2:319-26.

43. Donati A, Recchia G, Cavallini G, Bergamini E. Effect of aging and anti-aging caloric restriction on the endocrine regulation of rat liver autophagy. J Gerontol A Biol Sci Med Sci. 2008;63:550-5.

44. Lipinski MM, Zheng B, Lu T, Yan Z, Py BF, Ng A, et al. Genome-wide analysis reveals mechanisms modulating autophagy in normal brain aging and in Alzheimer's disease. Proc Natl Acad Sci. 2010;107:14164-9.

45. Harrison DE, Strong R, Sharp ZD, Nelson JF, Astle CM, Flurkey K, et al. Rapamycin fed late in life extends lifespan in genetically heterogeneous mice. Nature. 2009;460:392-5.

46. Choi AM, Ryter SW, Levine B. Autophagy in human health and disease. N Engl J Med. 2013;368:651-62.

47. Velarde MC, Flynn JM, Day NU, Melov S, Campisi J. Mitochondrial oxidative stress caused by Sod2 deficiency promotes cellular senescence and aging phenotypes in the skin. Aging (Albany NY). 2012;4:3-12.

48. Schapira AH, Gegg M. Mitochondrial contribution to Parkinson's disease pathogenesis. Parkinsons Dis. 2011;2011:159160.

49. Lin MT, Beal MF. Mitochondrial dysfunction and oxidative stress in neurodegenerative diseases. Nature. 2006;443:787-95.

50. de Magalhães JP, Curado J, Church GM. Meta-analysis of age-related gene expression profiles identifies common signatures of aging. Bioinformatics. 2009;25:875-81.

51. Zahn JM, Poosala S, Owen AB, Ingram DK, Lustig A, Carter A, et al. AGEMAP: a gene expression database for aging in mice. PLoS Genet. 2007;3:e201.

52. Liu LF, Shen WJ, Ueno M, Patel S, Kraemer FB. Characterization of agerelated gene expression profiling in bone marrow and epididymal adipocytes. BMC Genomics. 2011;12:212.
53. Hamatani T, Falco G, Carter MG, Akutsu H, Stagg CA, Sharov AA, et al. Age-associated alteration of gene expression patterns in mouse oocytes. Hum Mol Genet. 2004;13:2263-78.

54. Reznick RM, Zong H, Li J, Morino K, Moore IK, Yu HJ, et al. Aging-associated reductions in AMP-activated protein kinase activity and mitochondrial biogenesis. Cell Metab. 2007:5:151-6.

55. Scarpulla RC. Nuclear control of respiratory gene expression in mammalian cells. J Cell Biochem. 2006;97:673-83.

56. Litonin D, Sologub M, Shi Y, Savkina M, Anikin M, Falkenberg M, et al. Human mitochondrial transcription revisited: only TFAM and TFB2M are required for transcription of the mitochondrial genes in vitro. J Biol Chem. 2010;285:18129-33.

57. Scarpulla RC. Transcriptional paradigms in mammalian mitochondrial biogenesis and function. Physiol Rev. 2008;88:611-38.

58. Lum JJ, DeBerardinis RJ, Thompson CB. Autophagy in metazoans: cell survival in the land of plenty. Nat Rev Mol Cell Biol. 2005;6:439-48.

59. Hardie DG. AMP-activated protein kinase: an energy sensor that regulates all aspects of cell function. Genes Dev. 2011;25:1895-908.

60. Bild DE, Bluemke DA, Burke GL, Detrano R, ez Roux AV, Folsom AR, et al. Multi-ethnic study of atherosclerosis: objectives and design. Am J Epidemiol. 2002;156:871-81.

61. Dunning MJ, Smith ML, Ritchie ME, Tavare S. beadarray: R classes and methods for Illumina bead-based data. Bioinformatics. 2007;23:2183-4.

62. Smyth GK, Michaud J, Scott HS. Use of within-array replicate spots for assessing differential expression in microarray experiments. Bioinformatics. 2005;21:2067-75.

63. Du P, Kibbe WA, Lin SM. lumi: a pipeline for processing Illumina microarray. Bioinformatics. 2008;24:1547-8.

64. Du P, Zhang X, Huang CC, Jafari N, Kibbe WA, Hou L, et al. Comparison of Beta-value and M-value methods for quantifying methylation levels by microarray analysis. BMC Bioinformatics. 2010;11:587.

65. Price AL, Patterson NJ, Plenge RM, Weinblatt ME, Shadick NA, Reich D. Principal components analysis corrects for stratification in genome-wide association studies. Nat Genet. 2006;38:904-9.

66. Fox CS, White CC, Lohman K, Heard-Costa N, Cohen P, Zhang Y, et al. Genome-wide association of pericardial fat identifies a unique locus for ectopic fat. PLoS Genet. 2012;8:e1002705.

67. Storey JD, Tibshirani R. Statistical significance for genomewide studies. Proc Natl Acad Sci U S A. 2003;100:9440-5.

68. Subramanian A, Tamayo P, Mootha VK, Mukherjee S, Ebert BL, Gillette MA, et al. Gene set enrichment analysis: a knowledge-based approach for interpreting genome-wide expression profiles. Proc Natl Acad Sci U S A. 2005;102:15545-50.

69. Abbas AR, Baldwin D, Ma Y, Ouyang W, Gurney A, Martin F, et al. Immune response in silico (IRIS): immune-specific genes identified from a compendium of microarray expression data. Genes Immun. 2005;6:319-31.

70. Cline MS, Smoot M, Cerami E, Kuchinsky A, Landys N, Workman C, et al. Integration of biological networks and gene expression data using Cytoscape. Nat Protoc. 2007;2:2366-82.

71. Diao H, Li X, Hu S. The identification of dysfunctional crosstalk of pathways in Parkinson disease. Gene. 2013;515:159-62.

72. Laemmli UK. Cleavage of structural proteins during the assembly of the head of bacteriophage T4. Nature. 1970;227:680-5.

73. Simoes A, Pereira D, Amaral J, Nunes A, Gomes S, Rodrigues P, et al. Efficient recovery of proteins from multiple source samples after trizol(R) or trizol(R)LS RNA extraction and long-term storage. BMC Genomics. 2013;14:181.

74. Warr MR, Shore GC. Unique biology of Mcl-1: therapeutic opportunities in cancer. Curr Mol Med. 2008;8:138-47.

75. Kojima S, Hyakutake A, Koshikawa N, Nakagawara A, Takenaga K. MCL-1 V, a novel mouse antiapoptotic MCL-1 variant, generated by RNA splicing at a non-canonical splicing pair. Biochem Biophys Res Commun. 2010;391:492-7.

76. Maurer U, Charvet C+, Wagman AS, Dejardin E, Green DR. Glycogen synthase kinase-3 regulates mitochondrial outer membrane permeabilization and apoptosis by destabilization of MCL-1. Mol Cell. 2006;21:749-60.

77. Domina AM, Vrana JA, Gregory MA, Hann SR, Craig RW. MCL1 is phosphorylated in the PEST region and stabilized upon ERK activation in viable cells, and at additional sites with cytotoxic okadaic acid or taxol. Oncogene. 2004;23:5301-15. 
78. Perciavalle RM, Stewart DP, Koss B, Lynch J, Milasta S, Bathina M, et al. Anti-apoptotic MCL-1 localizes to the mitochondrial matrix and couples mitochondrial fusion to respiration. Nat Cell Biol. 2012;14:575-83.

79. Huang $\mathrm{CR}$, Yang-Yen HF. The fast-mobility isoform of mouse Mcl-1 is a mitochondrial matrix-localized protein with attenuated anti-apoptotic activity. FEBS Lett. 2010;584:3323-30.

80. Rosseel Y. Lavaan: an R package for structural equation modeling. J Stat Software. 2012;48:1-36.

Submit your next manuscript to BioMed Central and take full advantage of:

- Convenient online submission

- Thorough peer review

- No space constraints or color figure charges

- Immediate publication on acceptance

- Inclusion in PubMed, CAS, Scopus and Google Scholar

- Research which is freely available for redistribution 